\title{
Hyaluronic Acid Nanoparticles-encapsulated Bacterial Genotoxin for Targeted Therapy of Radioresistant Prostate Cancer
}

\section{Yu-An Chen}

UT Southwestern: The University of Texas Southwestern Medical Center

\section{Yi-Ru Lai}

Chang Gung University

\section{Hui-Yu Wu}

Chang Gung University

\section{Yen-Ju Lo}

Chang Gung University

\section{Yu-Fang Chang}

Chang Gung University

\section{Chiu-Lien Hung}

Industrial Technology Research Institute

\section{Chun-Jung Lin}

UT Southwestern: The University of Texas Southwestern Medical Center

\section{U-Ging Lo}

UT Southwestern: The University of Texas Southwestern Medical Center

Ho Lin

National Chung Hsing University

Jer-Tsong Hsieh

UT Southwestern: The University of Texas Southwestern Medical Center

\section{Cheng-Hsun Chiu}

Chang Gung Memorial Hospital

\section{Yu-Hsin Lin}

National Yang-Ming University

Chih-Ho Lai ( $\square$ chlai@mail.cgu.edu.tw)

Chang Gung University https://orcid.org/0000-0001-7145-784X

\section{Research}

Keywords: Cytolethal distending toxin, hyaluronic acid, CD44, nanoparticles, radioresistant 
Posted Date: December 1st, 2020

DOI: https://doi.org/10.21203/rs.3.rs-113497/v1

License: (c) (i) This work is licensed under a Creative Commons Attribution 4.0 International License. Read Full License 


\section{Abstract}

Background: Prostate cancer (PCa) is one of the most commonly diagnosed cancers in men and usually becomes refractory because of recurrence and metastasis. CD44, a transmembrane glycoprotein, serves as a receptor for hyaluronic acid (HA) and has been found to be abundantly expressed in cancer stem cells (CSCs) that often exhibit a radioresistant phenotype. Cytolethal distending toxin subunit B (CdtB), produced by Campylobacter jejuni, is a genotoxin acts as a type I deoxyribonuclease (DNase I), which is responsible for creating DNA double-strand breaks (DSBs). Nanoparticles loaded with antitumor drugs and specific ligands that recognize cancerous cell receptors are promising methods to overcome the therapeutic challenges.

Results: In this study, PCa cells with DOC-2/DAB2 interactive protein (DAB2IP) knockdown were employed to imitate the CSC with radioresistant phenotype. We further prepared HA-decorated nanoparticlesencapsulated CdtB (HA-CdtB-NPs) and investigated the targeted therapeutic activity in radioresistant PCa cells. Our results showed that HA-CdtB-NPs sensitized radioresistant PCa cells by enhancing DSB and causing G2/M cell-cycle arrest, without affecting the normal prostate epithelial cells. These results demonstrate that HA-CdtB-NPs possess maximum target-specificity and delivery efficiency of CdtB into the nucleus, and enhance the effect of radiation in radioresistant PCa cells.

Conclusions: These findings indicate that HA-loaded CdtB nanoparticles exert target-specificity accompanied with radiomimetic activity, which can be developed as an effective agent for overcoming radioresistance in $\mathrm{PCa}$.

\section{Background}

Prostate cancer $(\mathrm{PCa})$ is a threatening malignancy that demonstrates an escalating trend in both the incidence and mortality rate worldwide in the recent years [1]. Patients may develop several non-specific symptoms such as urinary retention, urinary hesitancy, nocturia, and hematuria [2]. Because of the high heterogeneity of $\mathrm{PCa}$, the relationship between certain symptoms and cancer development is rather weak and undefined [3]. However, attributed to the advancement in diagnostic tests, more people opt for clinical management during the early phases. Treatment strategies for PCa include surgery, chemotherapy, hormone therapy, and radiation therapy [4]. Among these, radiation therapy is considered as an effective remedy for localized cancers, which improves prognosis when combined with other appropriate treatment modalities $[5,6]$. Therefore, the unpredictable emergence of radioresistance may result in a setback for successful treatments. Even worse, development of radioresistance often leads to tumor metastasis or relapse [7]. To overcome this problem, a radiation sensitizer is likely to be the solution.

Cytolethal distending toxin (CDT), a genotoxin produced by Campylobacter jejuni (C. jejuni), causes DNA double-strand breaks (DSBs) [8]. CDT comprise three subunits: CdtA, CdtB, and CdtC, which is encoded by the consecutive genes $c d t A, c d t B$, and $c d t C$, respectively [9]. The working subunit, $C d t B$, acts as a DNase I, which is responsible for creating DSB, cell cycle arrest at the G2/M phase [10], thereby inducing cell 
distension and ultimately cell death [11]. However, CdtB cannot traverse the cell membrane alone and therefore, its entry into the cytoplasm requires the binding of CdtA and CdtC to the cell membrane. With the help of the nuclear localization signal (NLS), CdtB is subsequently translocated into the nucleus [12]. Given the similarity of CDT with radiation, wherein both elicit cell death by causing DSBs, it is highly possible for $\mathrm{CDT}$ to be an ideal radiation sensitizer.

Although CDT appears to be a promising cure for radioresistant PCa, the specific delivery of CDT to PCa cells remains a critical issue. This challenging task can be overcome by utilizing the CD44-overexpressing nature of PCa cells [13]. CD44 is a transmembrane glycoprotein which mediates extracellular adhesion and signal transduction [14]. In addition, CD44 has been involved in tumor progression and metastasis, drug resistance, and radiation resistance of cancer cells $[15,16]$. Several studies have demonstrated that using hyaluronic acid (HA)-decorated nanoparticles to encapsulate various therapeutic drugs could effectively target CD44-overexpressing cells and subsequently deliver the encapsulated agents into those cells [17-21]. HA, also known as hyaluronan, is a naturally occurring non-sulfated glycosaminoglycan, with characteristics such as biocompatibility, non-immunogenicity, and biodegradability, making it an ideal material for targeting $\mathrm{CD} 44^{+}$cancer cells [21].

In this study, HA-decorated nanoparticles-encapsulated CdtB (HA-CdtB-NPs) were developed and extensively studied for their characteristics, including the particle size, loading efficiency, and zetapotential. PCa cells with DOC-2/DAB2 interactive protein (DAB2IP) knockdown were chosen to imitate the cancer stem cells (CSC) with radioresistant phenotype [22]. After treating PC3 DAB2IP-knockdown (PC3KD) cells with HA-CdtB-NPs, the drug efficacy, cytotoxicity, and delivery efficiency were assessed.

\section{Materials And Methods}

\section{Antibodies and reagents}

Antibodies against cleaved-PARP was purchased from Proteintech (Chicago, IL). Antibodies specific to phospho-H2AX and phospho-CHK2 were purchased from Cell Signaling (Danvers, MA). Antibodies against $\beta$-actin, proliferating cell nuclear antigen (PCNA), and rabbit horseradish peroxidase (HRP)conjugated antibody were purchased from Santa Cruz Biotechnology (Santa Cruz, CA). Antibody against CD44 was purchased from Novus Biologicals (Centennial, CO). Other reagents and chemicals were purchased from Sigma-Aldrich (St. Louis, MO).

\section{Purification of CDT subunits}

Recombinant His-tagged CdtA, CdtB and CdtC subunits were constructed as described in our previous study [23]. A single colony of Escherichia coli BL21-DE3 containing $c d t A, c d t B, c d t C$ expression plasmids, respectively, were picked and cultured in LB broth with ampicillin $(100 \mu \mathrm{g} / \mathrm{mL})$ at $37^{\circ} \mathrm{C}$. E. coli harboring $c d t A, c d t B, c d t C$ recombinant plasmids were then induced by isopropyl $\beta$-D-1-thiogalactopyranoside (IPTG) at $37^{\circ} \mathrm{C}$ for $5 \mathrm{~h}$ for which containing $c d t A$ and $c d t B$, and at $16^{\circ} \mathrm{C}$ for $16 \mathrm{~h}$ for which containing $c d t C$, 
respectively. Recombinant proteins were purified by metal affinity chromatography (Clontech, CA, USA) and analyzed by SDS-PAGE and western blot assay.

\section{Preparation of HA-decorated nanoparticleencapsulated CdtB}

Recombinant CdtB was purified according to the protocol as described above. To prepare HA-decorated nanoparticle-encapsulated $\mathrm{CdtB}$, a simple ionic gelation with magnetic was stirred at room temperature. The determination of the optimal preparation conditions was performed by examining different proportions of HA/arginine-chitosan (HA/Arg-CS). In brief, aqueous HA (4.00 mg/mL, $0.50 \mathrm{~mL}$ ) was added into aqueous Arg-CS with various concentrations (1.00, 2.00, 3.00 or $4.00 \mathrm{mg} / \mathrm{mL}, 0.50 \mathrm{~mL}$ ), and then the solutions were gently shaken for $30 \mathrm{~min}$ at $37^{\circ} \mathrm{C}$. After collecting the prepared HA/Arg-CS NPs produced by centrifugation, a Zetasizer instrument was used to measure the particle size, polydispersity index, and zeta potentials. Meanwhile, the NP suspension was placed onto a mesh copper grid and positively stained with osmium tetroxide to observe their morphology under transmission electron microscopy.

Next, different concentrations of CdtB were encapsulated in nanoparticles to determine the optimal condition for preparing CdtB-loaded HA/Arg-CS NPs. CdtB dissolved in deionized water $(3.00,2.00,1.00$ $\mathrm{mg} / \mathrm{mL} ; 0.25 \mathrm{~mL})$ was mixed with HA solution $(8.00 \mathrm{mg} / \mathrm{mL} ; 0.25 \mathrm{~mL})$ to form CdtB/HA aqueous mixed solutions $(1.50: 4.00,1.00: 4.00$, and $0.50: 4.00 \mathrm{mg} / \mathrm{mL}, 0.50 \mathrm{~mL})$. The CdtB/HA solutions $(0.50 \mathrm{~mL})$ were added to the optimal concentration of Arg-CS in deionized water $(0.50 \mathrm{~mL})$ and stirred at $37^{\circ} \mathrm{C}$ for $30 \mathrm{~min}$. After centrifugation, the amount of free $\mathrm{CdtB}$ in the supernatant was detected by a protein concentration assay (Bio-Rad, CA, USA) and protein-loading efficiency of the NPs calculated from the following equation:

Loading efficiency $=\frac{\text { total } \mathrm{CdtB} \text { protein }- \text { free } \mathrm{CdtB} \text { protein }}{\text { total } \mathrm{CdtB} \text { protein }} \times 100 \%$

\section{Cell culture}

Human prostate epithelial cell line (PZ-HPV-7) was derived from the peripheral zone of a benign prostate and cultured as described previously [24]. The methods that used to knockdown endogenous DOC2/DAB2 interactive protein (DAB2IP) in PCa cells were described previously [25]. PC3 knockdown DAB2IP (PC3-KD) cells were cultured in Roswell Park Memorial Institute (RPMI) 1640 medium (Hyclone, Logan, UT) with $5 \%$ fetal bovine serum (FBS) (Hyclone) containing puromycin $(0.4 \mu \mathrm{g} / \mathrm{mL}), 1 \times$ penicillin/streptomycin, and $\mathrm{G} 418(800 \mu \mathrm{g} / \mathrm{mL})$ in the environment of $37^{\circ} \mathrm{C}$ and $5 \% \mathrm{CO}_{2}$.

\section{Analysis of cell viability}

PC3-KD cells were seeded in 96-well plates and treated with $0 \mathrm{nM}, 10 \mathrm{nM}, 50 \mathrm{nM}, 100 \mathrm{nM}, 200 \mathrm{nM}, 500$ $\mathrm{nM}$, and 1,000 nM of HA/Arg-CS (HA-NPs), CDT holotoxin and HA-CdtB-NPs, respectively. After incubation 
for $48 \mathrm{~h}$, cells were treated with $100 \mu \mathrm{L}$ of $0.5 \mathrm{mg} / \mathrm{mL}$ 3-(4,5-dimethylthiazol-2-yl)-2,5-diphenyltetrazolium bromide (MTT) solution in $37^{\circ} \mathrm{C}$ for $2 \mathrm{~h}$. Cell viability were analyzed by measuring the ability of viable cells reducing MTT (Sigma-Aldrich) to formazan [26].

\section{HA-CD44 localization and DNA double strand break (DSB) by immunofluorescence}

PC3-KD cells were seeded onto cover glasses and incubated for $24 \mathrm{~h}$. For DSB analysis, cells were first treated with $2 \mathrm{~Gy}$ ionizing radiation (IR), then exposed to $200 \mathrm{nM}$ of HA-NPs, CDT holotoxin and HA-CdtBNPs, respectively. Cells were subsequently fixed with $1 \%$ paraformaldehyde for $1 \mathrm{~h}$, permeabilized by $0.1 \%$ triton X-100 for 15 min. For HA-CD44 colocalization assay, cells were then treated with primary antibody against CdtB. For DSB analysis, cells were treated with anti-pH2AX and anti-53BP1 at the dilution of $1: 200$, followed by treatment with secondary antibody at the dilution of 1:500 and 4',6-diamidino-2phenylindole DAPI $(0.2 \mu \mathrm{g} / \mathrm{mL})$. The stained cells were observed using Laser Scanning Confocal Microscope (LSM780, ZEISS, Germany).

\section{Analysis of CdtB in the nuclear extraction}

PC3-KD cells were treated with 100 nM HA-NPs, CDT holotoxin, and HA-CdtB-NPs, followed by incubation for $0,0.5,1,3$, and $6 \mathrm{~h}$. The nuclear proteins were isolated using a nuclear extraction kit (Pierce, Rockford, IL), as described previously [23]. CdtB level in the nuclear fraction was then analyzed by western blot assay.

\section{Western blot analysis}

PC3-KD cells were seeded onto $60 \mathrm{~mm}$ dish and incubated in $37^{\circ} \mathrm{C}$ for $24 \mathrm{~h}$, then treated with medium (mock), IR, HA-NPs accompanied by IR, CDT holotoxin accompanied by IR, and HA-CdtB-NPs accompanied by IR, respectively, for $24 \mathrm{~h}$ and $48 \mathrm{~h}$. Cell lysates were centrifuged at 12,000 rpm for $20 \mathrm{~min}$ at $4^{\circ} \mathrm{C}$. After quantification, samples were resolved by $12 \%$ SDS-PAGE and transferred onto polyvinylidene difluoride membranes (PVDF, Millipore, MA). The PVDF membrane was blocked and probed with primary antibodies against (ADP-Ribose) P polymerase (PARP), pH2AX, pATM, pCHK2, and $\beta$-actin in the condition of gentle shaking. After an overnight incubation at $4^{\circ} \mathrm{C}$, the membrane was then probed with horseradish peroxidase-conjugated secondary antibody (Millipore) in room temperature for $1 \mathrm{~h}$. The proteins of interests were detected using ECL Western Blotting Detection Reagents (GE Healthcare, Piscataway, NJ, USA) and were visualized using an Azure c400 system and AzureSpot Analysis Software (Azure Biosystems, USA) by following the manufacturer's instructions.

\section{Cell cycle analysis by flow cytometry}

PC3-KD cells were incubated with ICRF-193 $(2 \mu \mathrm{g} / \mathrm{mL})$, HA-CdtB-NPs (50 nM,100 nM, and $200 \mathrm{nM})$, and HA-NPs alone for $24 \mathrm{~h}$. The cells were washed with PBS, centrifuged for 1,000 rpm in room temperature for $5 \mathrm{~min}$. Subsequently, the prepared cells were resuspended in hypotonic buffer $(0.2 \mathrm{mg} / \mathrm{mL}$ RNase A, $20 \mu \mathrm{g} / \mathrm{mL}$ propidium iodide, and $0.1 \%$ Triton $\mathrm{X}-100$ ). Analysis of cell cycle were performed by flow cytometry (Becton Dickinson, CA). 


\section{Comet assay}

Experiment protocols followed instructions by Trevigen CometAssay ${ }^{\circledR}$ Kit (Trevigen, Gaithersburg, MD, USA). PC3-KD cells were seeded in $60 \mathrm{~mm}$ dish and respectively treated with medium (mock), HA-NPs, CDT holotoxin, and HA-CdtB-NPs with three of the latter accompanied with IR. After incubation for $24 \mathrm{~h}$, PC3-KD cells $\left(1 \times 10^{5} / \mathrm{mL}\right)$ were first mixed with LMAgarose with the proportion of 1:10 and placed onto CometSlide ${ }^{\mathrm{TM}}$ with complete coverage of sample area, then incubated the slide at $4^{\circ} \mathrm{C}$ in the dark for 30 min. Cells were then immersed with $4^{\circ} \mathrm{C}$ lysis solution at $4^{\circ} \mathrm{C}$ for $30-60 \mathrm{~min}$ and followed by alkaline unwinding solution at $4^{\circ} \mathrm{C}$ in the dark for $1 \mathrm{~h}$. CometSlide ${ }^{\mathrm{TM}}$ were placed in alkaline electrophoresis solution and set power supply to 21 volts then applied voltage for 30 min and washed by distillationdistillation $\mathrm{H}_{2} \mathrm{O}\left(\mathrm{ddH}_{2} \mathrm{O}\right)$ and $70 \%$ alcohol. After CometSlide ${ }^{\mathrm{TM}}$ were dried and stained by $\mathrm{CYBR}^{\circledR}$ Gold, cells were observed using Leica CTR 4000 (Leica, Germany).

\section{Statistical analysis}

The analysis among multiple groups was carried out by means of one-way analysis of variance. The post-hoc test was used to measure the statistical significance of differences between two groups. Statistical analyses and chart drafting were carried out using the GraphPad Prism 6 software (GraphPad Software Inc, CA, USA).

\section{Results}

\section{Preparation and characterization of CDT and HA-CdtB-NPs}

Purified recombinant CDT subunits and synthesized HA-CdtB-NPs were analyzed by SDS-PAGE (Fig. 1A and 1B). The NPs were constructed with negatively charged HA and positively charged arginine-chitosan (Arg-CS). As shown in Table 1, HA:Arg-CS used in distinct concentrations (2.0:0.5, 2.0:1.0, and 2.0:2.0 by $\mathrm{mg} / \mathrm{mL}$ ) formed complexes on the nanometer scale, with the exception of the concentration 2.0:1.5 $\mathrm{mg} / \mathrm{mL}$. The diameters of the prepared NPs were in the range of 220-300 nm (Table 1 and Fig. 1C), and the negatively or positively charged values were dependent on the relative concentrations of $\mathrm{HA}$ and Arg$\mathrm{CS}$. When the amount of the positively charged Arg-CS sufficiently exceeded that of the negatively charged HA (HA:Arg-CS = 2.0:2.0 mg/mL), the NPs had Arg-CS exposed on their surfaces and thus, had a positive surface charge. In contrast, when the amount of the negatively charged HA significantly exceeded that of the positively charged Arg-CS (HA:Arg-CS $=0.5: 2.0$ or $1.0: 2.0$ by $\mathrm{mg} / \mathrm{mL}$ ), some of the excessive HA molecules were entangled onto the surfaces of the obtained NPs. Thus, the resulting NPs displayed a negative surface charge. The HA/Arg-CS concentration of $2.0: 0.5 \mathrm{mg} / \mathrm{mL}$ produced the mean particle size of $225.64 \pm 14.65 \mathrm{~nm}$ with a maximum negative zeta potential of $-38.79 \pm 4.51 \mathrm{mV}(\mathrm{n}=5)$. Therefore, the NPs prepared with this specific composition were used for the following studies.

In order to prepare the CdtB-encapsulated NPs, various concentrations of CdtB were mixed with HA/ArgCS encapsulated mixture solution. As shown in Table 2, the CdtB:HA:Arg-CS in distinct concentrations 
showed a mean size range of $250-300 \mathrm{~nm}$, with different negative potential values. When the concentration of CdtB:HA:Arg-CS is $0.75: 2.00: 0.50 \mathrm{mg} / \mathrm{mL}$, the loading efficiency of CdtB in NP is $48.97 \pm$ $5.12 \%$, its particle size is $273.78 \pm 17.37 \mathrm{~nm}$, zeta potential value is $-39.89 \pm 9.65 \mathrm{mV}$. In addition, the polydispersity index of NPs measured using dynamic light scattering (DLS) revealed a narrow distribution (polydispersity index: $0.27 \pm 0.06$ ) and the morphology of the prepared NPs along with CdtBencapsulated NPs remained spherical and smooth-shaped on transmission electron microscopy observation (Fig. 1D). Therefore, the HA-decorated NPs encapsulating CdtB with a negative surface charge at a CdtB:HA:Arg-CS concentration of $0.75: 2.00: 0.50 \mathrm{mg} / \mathrm{mL}$ were used for the remaining studies.

\section{HA-CdtB-NPs induce radioresistant PCa cell death}

Loss of DOC-2/DAB2 interactive protein (DAB2IP) expression in PCa cells leads to the development of radioresistance [25]. We therefore utilized DAB2IP-knockdown PCa cells (PC3-KD) as the research platform to investigate the therapeutic effects of HA-CdtB-NPs on radioresistant PCas. We first analyzed the CD44 expression in PCa cells and normal prostate epithelial cells. As shown in Fig. S1, higher CD44 expression was observed in PC3-KD cells than in PZ-HPV-7 cells. For evaluating the ability of HA-CdtBNPs to induce PCa cell death, we performed an MTT assay to analyze the effect of HA-NPs, CDT holotoxin, and HA-CdtB-NPs on the cell viability of the PC3-KD cells and PZ-HPV-7 cells. To determine the most ideal condition for the following experiments, we treated PC3-KD cells with various concentrations of the HA-CdtB-NPs and found that the viable cell numbers dropped to approximately half at a concentration of $100 \mathrm{nM}$ (Fig. 2A). Meanwhile, CDT holotoxin treatment also exhibited a reduction effect in a concentration-dependent manner; however, it was less effective than HA-CdtB-NPs. Furthermore, it was denoted that HA-NPs barely affected both PC3-KD and PZ-HPV-7 cells (Fig. 2B). Most importantly, PZ-HPV-7 cells did not show any evident response to HA-CdtB-NPs treatment, which indicated that HACdtB-NPs are able to specifically target PCa cells.

\section{Delivery of HA-CdtB-NPs in PCa cells}

To assess the delivery efficiency of HA-CdtB-NPs into PCa cells, we treated PC3-KD cells with HA-CdtB-NP for different time periods and observed the colocalization of CD44 and HA. As shown in Fig. 3A, CD44-HA colocalization could be noted at $0.5,1$, and $3 \mathrm{~h}$, with the peak level of colocalization observed at $1 \mathrm{~h}$. In addition, we performed a western blot analysis for checking the nuclear translocation of CdtB and the expression levels were also quantified (Fig. 3B, C). The highest expression of CdtB in the nucleus was observed at $6 \mathrm{~h}$ after HA-CdtB-NPs treatment. Interestingly, PC3-KD cells treated with HA-CdtB-NPs demonstrated significantly higher expression of CdtB in the nucleus than those treated with CDT holotoxin. The results showed that HA-CdtB-NPs attached to PC3-KD cells primarily at $1 \mathrm{~h}$ after the treatment, and at $6 \mathrm{~h}$, most CdtB entered the nucleus. Our results indicated that HA-CdtB-NPs manifested better delivery efficiency of CdtB into the nucleus than CDT holotoxin, which corresponded with the cell viability assay.

\section{HA-CdtB-NPs enhance IR-induced DSB}


To examine whether HA-CdtB-NPs regain the sensitivity of PC3-KD cells toward IR, three different procedures that allow the visualization of DSBs were performed, and four conditions were included: mock treatment, HA-NPs/IR cotreatment, CDT holotoxin/IR cotreatment, and HA-CdtB-NPs/IR cotreatment. First, in the comet assay, comet-like structures were observed in the cells for all treatment conditions except for the mock treatment (Fig. 4A). Nevertheless, the HA-CdtB-NPs/IR cotreatment displayed a brighter and longer tail than the other treatments. Second, expression levels of $\mathrm{Y}-\mathrm{H} 2 \mathrm{AX}, \mathrm{p}-\mathrm{CHK} 2$, and cleaved-PARP were analyzed using western blot (Fig. 4B). HA-NPs/IR cotreatment manifested almost similar low expression as the mock treatment. Although the $\mathrm{Y}-\mathrm{H} 2 \mathrm{AX}$ and $\mathrm{p}-\mathrm{CHK} 2$ expressions in CDT holotoxin/IR and HA-CdtB-NPs/IR treatments are similar, expression levels of $\mathrm{Y}$-H2AX, p-CHK2, and cleaved-PARP are still the highest in HA-CdtB-NPs/IR treatment among all the treating conditions. Finally, as shown by the immunofluorescence staining, the extent of DSB is indicated using the number of foci of $\mathrm{y}-\mathrm{H} 2 \mathrm{AX}$ and 53BP1 (Fig. 5). Similar to the previous results, the foci were observable in all treatment conditions except for mock-control, and HA-CdtB-NPs/IR cotreatment solely had maximum foci formation. These results reveal that HA-CdtB-NPs were the most effective and could synergistically enhance IR-induced DSB in radioresistant $\mathrm{PCa}$ cells.

\section{HA-CdtB-NPs induce cell cycle arrest}

Since DSB elicits the spontaneous activation of DNA damage response, which is likely to arrest the cell cycle, we further characterized the effect of HA-CdtB-NPs on the cell cycle distribution. For the control group and HA-NPs treatment group, merely $6.29 \%$ and $6.25 \%$ of cells were arrested at the G2/M phase (Fig. 6A, B). ICRF-193 treatment served as a positive control, which arrested $89.82 \%$ of cells at the G2/M phase. An apparent increase in the population in the G2/M phase was detected with HA-CdtB-NPs treatment; moreover, the increase in HA-CdtB-NPs concentration also increased the population at the G2/M phase. These results demonstrate that HA-CdtB-NPs possessed a better activity that induced DSBs and cell cycle arrest at the $\mathrm{G} 2 / \mathrm{M}$ phase.

\section{Discussion}

Radiotherapy has long been a powerful strategy against cancer cell proliferation; however the malignant cancer cells often find ways to bypass cell death [7, 27-29]. The utility of combinatorial treatment modalities, specifically radiosensitizers in cancer therapy, have been investigated in the early 1970s [30]. Recently, nanotechnology has pioneered a bright road towards the development of innovative cancer therapy platform [31,32]. Owing to the possibility of modification of nanoparticle composition and surface coating, the functions and specificity of nanoparticles could be more flexibly customized [33-35].

In our previous study, we have developed nanoparticles of HA and polyethylene glycol-gelatin (PEGgelatin) to specifically target CD44 ${ }^{+}$PCa cells [36]. It has been revealed that CD44 expressing cells exhibit stem cell-like properties [37, 38], and could be an ideal target for selectively killing CSC [39]. Mounting evidence has suggested the likelihood of CSC involvement in radioresistance [40-42], supporting the utility of CSC-targeted radiosensitizer to improve the efficacy of radiotherapy. 
Many bacterial toxins have been extensively studied for their promising clinical applications [43-47]. A previous study has shown that human gingival squamous carcinoma cell line Ca9-22 transfected with Actinobacillus actinomycetemcomitans-CdtB expressing plasmid, manifested $\mathrm{G} 2$ phase arrest and cell growth inhibition [48]. Additionally, in our previous work, chitosan/heparin nanoparticle-encapsulating CdtB was proved to be effective in causing G2/M phase cell cycle arrest in gastric cancer cells and finally induced cell death [49]. The potential of CDT as a radiosensitizer also has been tested in PCa models [50]. As described before, CDT exerts a similar effect as radiation via CdtB entry into the nucleus, but with the precondition of CdtA and CdtC binding to the cell surface. To minimize the size of the particles for better cellular uptake efficiency and tumor permeability, nanoparticles allowing intracellular delivery were designed, and only CdtB (without CdtA and $\mathrm{CdtC}$ ) was purified and encapsulated into the nanoparticles. In addition, PEG-gelatin nanoparticles possess the preferable advantages such as biocompatibility, biodegradability and cost-effectiveness $[51,52]$. Gelatin is a denatured protein obtained from animal structural collagen [53], which can properly form a complete structure with CdtB; in addition, the PEGmodification provides the protection against enzymatic damage and mononuclear phagocytosis system [54], which allows a sustained circulation.

In this study, we attempted to analyze the potential of CDT as a radiosensitizer, and further exploited progressive nanotechnology to develop a novel combinatorial treatment for PCa. To determine the binding activity of HA-CdtB-NPs, we visualized the localization of HA-CdtB-NPs using immunofluorescence and found that most HA-CdtB-NPs attached to the target cells as early as $1 \mathrm{~h}$ after treatment. Moreover, the amount of CdtB entry into the cytosol and subsequently the cell nuclei with the HA/PEG-gelatin delivery system was the highest at $6 \mathrm{~h}$ after treatment. Notably, the results also indicated that HA-CdtB-NPs have a better CdtB delivery efficiency than CDT holotoxin. Hence, CdtB has the potential to cause cancer cell death by directing DSB, which makes it an effective radiosensitizer.

The enhancement of radiosensitivity was further examined by the combined effect of HA-CdtB-NPs and IR. DSB formation and the phosphorylation of downstream proteins such as $\mathrm{CHK} 2$ and $\mathrm{y}-\mathrm{H} 2 \mathrm{AX}$ confirmed the activation of ATM-dependent DNA damage signaling pathway [55]. PCa cells treated with IR showed positive results, while HA-CdtB-NPs/IR presented the most synergistic effect. Furthermore, after $24 \mathrm{~h}$ treatment with HA-CdtB-NPs, a large proportion of PCa cells were paused at the G2/M phase, which has been revealed to be the most vulnerable stage for radiation [56-58].

Although our results show that HA-CdtB-NPs possess the potent therapeutic effect in radioresistant PCa cells, further investigations are required for a more realistic medical application. For instance, even though previous research showed that HA has an equivalent cancer cell cytotoxicity as that of anti-CD44 antibodies, CD44 does not serve as the only receptor for HA and it may not be a specific biomarker for PCa cells [39]. Therefore, in vivo studies aimed at the analysis of biodistribution, circulation time, antitumor activity and side effects of the HA-CdtB-NPs should be performed.

\section{Conclusion}


In this study, we developed an HA-based nanoparticle delivery system to carry CdtB into PCa cells. Our results demonstrated that HA-CdtB-NPs possess activity similar to CDT holotoxin, but with superior advantages. The potential effects include maximum target-specificity and delivery efficiency of CdtB into the nucleus, and enhancement of the effect of IR in radioresistant PCa cells. Our results provide a novel strategy to employ HA-nanoparticles encapsulated with CdtB as a radiation sensitizer, which can be developed as a potent therapeutic agent for radioresistant PCa.

\section{Declarations}

\section{Acknowledgments}

The authors would like to thank the editor and reviewers for the editorial assistance and their valuable comments. The authors sincerely appreciate the assistance for analyzing confocal microscopy (Microscopy Center, Chang Gung University, Taoyuan, Taiwan).

\section{Funding}

This study is supported by Taiwan Ministry of Science and Technology (108-2911-I-005-509, 109-2911-I005-503, 109-2320-B-182-025-MY3, and 109-2320-B-182-029-MY3), Chang Gung Memorial Hospital (CMRPD1I0061-3, CMRPD1J0021-3, CMRPD1K0361, and BMRPE90), and Tomorrow Medical Foundation.

\section{Competing interests}

The authors declare that they have no competing interests.

\section{Author's contributions}

Conception or design of this work: $\mathrm{HL}, \mathrm{JTH}, \mathrm{CHC}, \mathrm{YHL}$, and CHL; Experimental study: CYC, YRL, HYW, YJL, and YFC; Data analysis and interpretation: $\mathrm{CLH}, \mathrm{CJL}$, and UGL; Writing the manuscript: CYC, YRL, CHC, $\mathrm{YHL}$, and $\mathrm{CHL}$; Final approval: all authors. ${ }^{\dagger} \mathrm{CYC}$ and $\mathrm{YRL}$ were equally contributed to this work.

\section{Consent for publication}

Not applicable.

\section{Availability of supporting data}

All the data and materials presented in the current study along with additional files are available.

\section{Author details}

${ }^{1}$ Graduate Institute of Biomedical Sciences, Department of Microbiology and Immunology, Chang Gung University, Taoyuan, Taiwan. ${ }^{2}$ Department of Life Sciences, National Chung Hsing University, Taichung, Taiwan. ${ }^{3}$ Department of Urology, University of Texas Southwestern Medical Center, Dallas, Texas, USA. 
${ }^{4}$ Targeted Drug and Delivery Technology Division, Biomedical Technology and Device Research Laboratories, Industrial Technology Research Institute, Hsinchu, Taiwan. ${ }^{5}$ Faculty of Pharmacy, Institute of Biopharmaceutical Sciences, Department and Institute of Pharmacology, Center for Advanced Pharmaceutics and Drug Delivery Research, National Yang-Ming University, Taipei, Taiwan. ${ }^{6}$ School of Medicine, Department of Medical Research, China Medical University and Hospital, Taichung, Taiwan.

${ }^{7}$ Department of Nursing, Asia University, Taichung, Taiwan. ${ }^{8}$ Molecular Infectious Disease Research Center, Department of Pediatrics, Chang Gung Memorial Hospital, Linkou, Taiwan.

\section{References}

1. Kimura T, Egawa S. Epidemiology of prostate cancer in Asian countries. Int J Urol. 2018;25:524-31.

2. Hamilton W, Sharp DJ, Peters TJ, Round AP. Clinical features of prostate cancer before diagnosis: a population-based, case-control study. Br J Gen Pract. 2006;56:756-62.

3. Tolkach Y, Kristiansen G. The heterogeneity of prostate cancer: a practical approach. Pathobiology. 2018;85:108-16.

4. Cooperberg MR, Broering JM, Carroll PR. Time trends and local variation in primary treatment of localized prostate cancer. J Clin Oncol. 2010;28:1117-23.

5. Baskar R, Lee KA, Yeo R, Yeoh KW. Cancer and radiation therapy: current advances and future directions. Int J Med Sci. 2012;9:193-9.

6. Incrocci L. Radiotherapy for prostate cancer and sexual health. Transl Androl Urol. 2015;4:124-30.

7. Chaiswing L, Weiss HL, Jayswal RD, Clair DKS, Kyprianou N. Profiles of radioresistance mechanisms in prostate cancer. Crit Rev Oncog. 2018;23:39-67.

8. Lara-Tejero M, Galan JE. A bacterial toxin that controls cell cycle progression as a deoxyribonuclease I-like protein. Science (New York, NY. 2000;290:354-7.

9. Pickett CL, Pesci EC, Cottle DL, Russell G, Erdem AN, Zeytin H. Prevalence of cytolethal distending toxin production in Campylobacter jejuni and relatedness of Campylobacter sp. cdtB gene. Infect Immun. 1996;64:2070-8.

10. Whitehouse CA, Balbo PB, Pesci EC, Cottle DL, Mirabito PM, Pickett CL. Campylobacter jejuni cytolethal distending toxin causes a G2-phase cell cycle block. Infect Immun. 1998;66:1934-40.

11. Lai CK, Chen YA, Lin CJ, Lin HJ, Kao MC, Huang MZ, et al. Molecular mechanisms and potential clinical applications of Campylobacter jejuni cytolethal distending toxin. Front Cell Infect Microbiol. 2016;6:9.

12. DiRienzo JM. Uptake and processing of the cytolethal distending toxin by mammalian cells. Toxins (Basel). 2014;6:3098-116.

13. Ni J, Cozzi PJ, Hao JL, Beretov J, Chang L, Duan W, et al. CD44 variant 6 is associated with prostate cancer metastasis and chemo-/radioresistance. Prostate. 2014;74:602-17.

14. Ponta H, Sherman L, Herrlich PA. CD44: from adhesion molecules to signalling regulators. Nat Rev Mol Cell Biol. 2003;4:33-45. 
15. Naor D, Sionov RV, Ish-Shalom D. CD44: structure, function, and association with the malignant process. Adv Cancer Res. 1997;71:241-319.

16. Xiao W, Graham PH, Power CA, Hao J, Kearsley JH, Li Y. CD44 is a biomarker associated with human prostate cancer radiation sensitivity. Clin Exp Metastasis. 2012;29:1-9.

17. Vangara KK, Liu JL, Palakurthi S. Hyaluronic acid-decorated PLGA-PEG nanoparticles for targeted delivery of SN-38 to ovarian cancer. Anticancer Res. 2013;33:2425-34.

18. Tran TH, Choi JY, Ramasamy T, Truong DH, Nguyen CN, Choi HG, et al. Hyaluronic acid-coated solid lipid nanoparticles for targeted delivery of vorinostat to CD44 overexpressing cancer cells. Carbohydr Polym. 2014;114:407-15.

19. Wang T, Hou J, Su C, Zhao L, Shi Y. Hyaluronic acid-coated chitosan nanoparticles induce ROSmediated tumor cell apoptosis and enhance antitumor efficiency by targeted drug delivery via CD44. J Nanobiotechnology. 2017;15:7.

20. Chiesa E, Dorati R, Conti B, Modena T, Cova E, Meloni F, et al. Hyaluronic acid-decorated chitosan nanoparticles for CD44-targeted delivery of everolimus. Int J Mol Sci. 2018;19.

21. Cai J, Fu J, Li R, Zhang F, Ling G, Zhang P. A potential carrier for anti-tumor targeted deliveryhyaluronic acid nanoparticles. Carbohydr Polym. 2019;208:356-64.

22. Yun EJ, Baek ST, Xie D, Tseng SF, Dobin T, Hernandez E, et al. DAB2IP regulates cancer stem cell phenotypes through modulating stem cell factor receptor and ZEB1. Oncogene. 2014.

23. Lin CD, Lai CK, Lin YH, Hsieh JT, Sing YT, Chang YC, et al. Cholesterol depletion reduces entry of Campylobacter jejuni cytolethal distending toxin and attenuates intoxication of host cells. Infection and immunity. 2011;79:3563-75.

24. Menon JU, Tumati V, Hsieh JT, Nguyen KT, Saha D. Polymeric nanoparticles for targeted radiosensitization of prostate cancer cells. J Biomed Mater Res A. 2015;103:1632-9.

25. Xie D, Gore C, Liu J, Pong RC, Mason R, Hao G, et al. Role of DAB2IP in modulating epithelial-tomesenchymal transition and prostate cancer metastasis. Proc Natl Acad Sci U S A. 2010;107:248590.

26. Chen YA, Tzeng DTW, Huang YP, Lin CJ, Lo UG, Wu CL, et al. Antrocin Sensitizes Prostate Cancer Cells to Radiotherapy through Inhibiting PI3K/AKT and MAPK Signaling Pathways. Cancers (Basel). 2018;11.

27. Schulz A, Meyer F, Dubrovska A, Borgmann K. Cancer stem cells and radioresistance: DNA repair and beyond. Cancers (Basel). 2019;11.

28. Jarosz-Biej M, Smolarczyk R, Cichon T, Kulach N. Tumor microenvironment as a "game changer" in cancer radiotherapy. Int J Mol Sci. 2019;20.

29. Kim W, Lee S, Seo D, Kim D, Kim K, Kim E, et al. Cellular stress responses in radiotherapy. Cells. $2019 ; 8$.

30. Fowler JF, Adams GE, Denekamp J. Radiosensitizers of hypoxic cells in solid tumors. Cancer Treat Rev. 1976;3:227-56. 
31. Kuncic Z, Lacombe S. Nanoparticle radio-enhancement: principles, progress and application to cancer treatment. Phys Med Biol. 2018;63:02TR1.

32. Jiang W, Chen J, Gong C, Wang Y, Gao Y, Yuan Y. Intravenous delivery of enzalutamide based on high drug loading multifunctional graphene oxide nanoparticles for castration-resistant prostate cancer therapy. J Nanobiotechnology. 2020;18:50.

33. Mirjolet C, Boudon J, Loiseau A, Chevrier S, Boidot R, Oudot A, et al. Docetaxel-titanate nanotubes enhance radiosensitivity in an androgen-independent prostate cancer model. Int $\mathrm{J}$ Nanomedicine. 2017;12:6357-64.

34. Wu M, Liu J, Hu C, Li D, Yang J, Wu Z, et al. Olaparib nanoparticles potentiated radiosensitization effects on lung cancer. Int J Nanomedicine. 2018;13:8461-72.

35. Hua L, Wang Z, Zhao L, Mao H, Wang G, Zhang K, et al. Hypoxia-responsive lipid-poly-(hypoxic radiosensitized polyprodrug) nanoparticles for glioma chemo- and radiotherapy. Theranostics. 2018;8:5088-105.

36. Huang WY, Lin JN, Hsieh JT, Chou SC, Lai CH, Yun EJ, et al. Nanoparticle targeting CD44-positive cancer cells for site-specific drug delivery in prostate cancer therapy. ACS Appl Mater Interfaces. 2016;8:30722-34.

37. Horta S, Agostinho AL, Mateus R, Pereira L, Pereira C, Capinha L, et al. Looking out for cancer stem cells' properties: the value-driving role of CD44 for personalized medicines. Curr Cancer Drug Targets. 2015;14:832-49.

38. Yan Y, Zuo X, Wei D. Concise review: emerging role of CD44 in cancer stem cells: a promising biomarker and therapeutic target. Stem Cells Transl Med. 2015;4:1033-43.

39. Morillas-Becerril L, Peta E, Gabrielli L, Russo V, Lubian E, Nodari L, et al. Multifunctional, CD44v6targeted ORMOSIL nanoparticles enhance drugs toxicity in cancer cells. Nanomaterials (Basel). 2020;10.

40. Peitzsch C, Kurth I, Kunz-Schughart L, Baumann M, Dubrovska A. Discovery of the cancer stem cell related determinants of radioresistance. Radiother Oncol. 2013;108:378-87.

41. Krause M, Dubrovska A, Linge A, Baumann M. Cancer stem cells: Radioresistance, prediction of radiotherapy outcome and specific targets for combined treatments. Adv Drug Deliv Rev. 2017;109:63-73.

42. Arnold CR, Mangesius J, Skvortsova, II, Ganswindt U. The role of cancer stem cells in radiation resistance. Front Oncol. 2020;10:164.

43. Frankel AE, Rossi P, Kuzel TM, Foss F. Diphtheria fusion protein therapy of chemoresistant malignancies. Curr Cancer Drug Targets. 2002;2:19-36.

44. Gurnev PA, Nestorovich EM. Channel-forming bacterial toxins in biosensing and macromolecule delivery. Toxins (Basel). 2014;6:2483-540.

45. Bachran C, Leppla SH. Tumor Targeting and Drug Delivery by Anthrax Toxin. Toxins (Basel). 2016;8.

46. Zahaf NI, Schmidt G. Bacterial Toxins for Cancer Therapy. Toxins (Basel). 2017;9. 
47. Ryou JH, Sohn YK, Hwang DE, Park WY, Kim N, Heo WD, et al. Engineering of bacterial exotoxins for highly efficient and receptor-specific intracellular delivery of diverse cargos. Biotechnol Bioeng. 2016;113:1639-46.

48. Iwanaga K, Tominaga K, Yamamoto K, Habu M, Maeda H, Akifusa S, et al. Local delivery system of cytotoxic agents to tumors by focused sonoporation. Cancer Gene Ther. 2007;14:354-63.

49. Lai CK, Lu YL, Hsieh JT, Tsai SC, Feng CL, Tsai YS, et al. Development of chitosan/heparin nanoparticle-encapsulated cytolethal distending toxin for gastric cancer therapy. Nanomedicine (Lond). 2014;9:803-17.

50. Lai CH, Chang CS, Liu HH, Tsai YS, Hsu FM, Yu YL, et al. Sensitization of radio-resistant prostate cancer cells with a unique cytolethal distending toxin. Oncotarget. 2014;5:5523-34.

51. Kaul G, Amiji M. Long-circulating poly(ethylene glycol)-modified gelatin nanoparticles for intracellular delivery. Pharm Res. 2002;19:1061-7.

52. Kaul G, Amiji M. Tumor-targeted gene delivery using poly(ethylene glycol)-modified gelatin nanoparticles: in vitro and in vivo studies. Pharm Res. 2005;22:951-61.

53. Sahoo N, Sahoo RK, Biswas N, Guha A, Kuotsu K. Recent advancement of gelatin nanoparticles in drug and vaccine delivery. Int J Biol Macromol. 2015;81:317-31.

54. Harris JM, Martin NE, Modi M. Pegylation: a novel process for modifying pharmacokinetics. Clin Pharmacokinet. 2001;40:539-51.

55. Bonner WM, Redon CE, Dickey JS, Nakamura AJ, Sedelnikova OA, Solier S, et al. GammaH2AX and cancer. Nat Rev Cancer. 2008;8:957-67.

56. Liu C, Nie J, Wang R, Mao W. The Cell Cycle G2/M Block Is an Indicator of Cellular Radiosensitivity. Dose Response. 2019;17:1559325819891008.

57. Luo Y, Chen X, Luo L, Zhang Q, Gao C, Zhuang X, et al. [6]-Gingerol enhances the radiosensitivity of gastric cancer via G2/M phase arrest and apoptosis induction. Oncol Rep. 2018;39:2252-60.

58. Lin S, Yan Y, Liu Y, Gao CZ, Shan D, Li Y, et al. Sensitisation of human lung adenocarcinoma A549 cells to radiotherapy by Nimotuzumab is associated with enhanced apoptosis and cell cycle arrest in the G2/M phase. Cell Biol Int. 2015;39:146-51.

\section{Tables}

Table 1. Characteristics of prepared NPs constructed with differential HA:Arg-CS concentrations. 


\begin{tabular}{|llll|}
\hline $\begin{array}{l}\text { HA:Arg-CS } \\
(\mathrm{mg} / \mathrm{mL})^{\dagger}\end{array}$ & $\begin{array}{l}\text { Mean particle } \\
\text { size }(\mathrm{nm})\end{array}$ & Polydispersity indices & Zeta potential value $(\mathrm{mV})$ \\
\hline $2.00: 0.50$ & $225.64 \pm 14.65$ & $0.23 \pm 0.08$ & $-38.79 \pm 4.51$ \\
\hline $2.00: 1.00$ & $287.76 \pm 37.78$ & $0.35 \pm 0.11$ & $-27.72 \pm 5.74$ \\
\hline $2.00: 1.50$ & $1165.98 \pm 325.87$ & $0.78 \pm 0.21$ & $-6.79 \pm 3.24$ \\
\hline $2.00: 2.00$ & $298.28 \pm 28.74$ & $0.19 \pm 0.12$ & $+26.47 \pm 2.95$ \\
\hline
\end{tabular}

${ }^{\dagger}$ Different HA:Arg-CS concentrations resulted in distinct particle sizes, zeta potential values, polydispersity indices of the prepared NPs $(n=5)$.

Table 2. Characteristics of HA-NPs encapsulating varying CdtB concentrations.

\begin{tabular}{|lllll|}
\hline $\begin{array}{l}\text { CdtB:HA:Arg-CS } \\
(\mathrm{mg} / \mathrm{mL})^{+}\end{array}$ & $\begin{array}{l}\text { Mean Particle } \\
\text { Size }(\mathrm{nm})\end{array}$ & $\begin{array}{l}\text { Polydispersity } \\
\text { indices }\end{array}$ & $\begin{array}{l}\text { Zeta Potential } \\
\text { Value }(\mathrm{mV})\end{array}$ & $\begin{array}{l}\text { CdtB Loading } \\
\text { Efficiency }(\%)\end{array}$ \\
\hline $0.75: 2.00: 0.50$ & $273.78 \pm 17.37$ & $0.27 \pm 0.06$ & $-38.89 \pm 9.65$ & $48.97 \pm 5.12$ \\
\hline $0.50: 2.00: 0.50$ & $261.43 \pm 18.95$ & $0.28 \pm 0.08$ & $-35.48 \pm 7.32$ & $45.79 \pm 6.51$ \\
\hline $0.25: 2.00: 0.50$ & $248.62 \pm 20.16$ & $0.21 \pm 0.09$ & $-35.13 \pm 4.83$ & $43.87 \pm 4.98$ \\
\hline
\end{tabular}

${ }^{\dagger}$ Different encapsulated CdtB concentrations resulted in distinct particle sizes, polydispersity indexes, zeta potentials, and CdtB-loading efficiencies of HA-NPs $(n=5)$.

\section{Figures}


A

kDa M CdtA CdtB CdtC

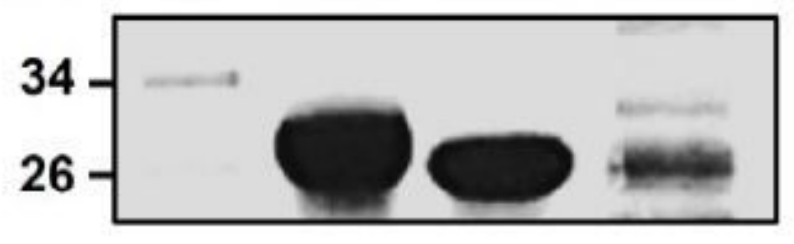

C

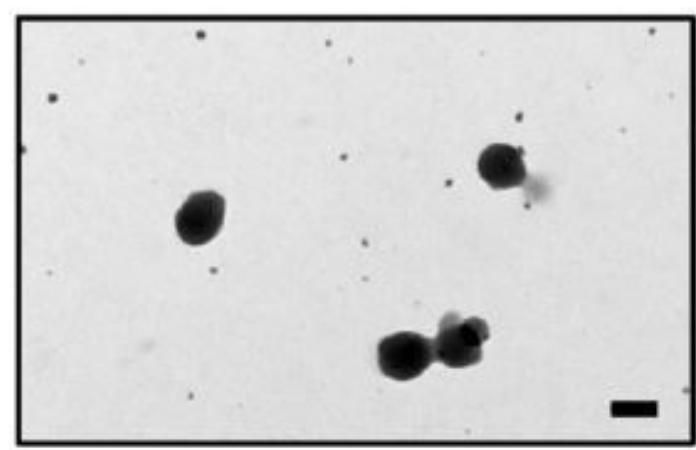

B
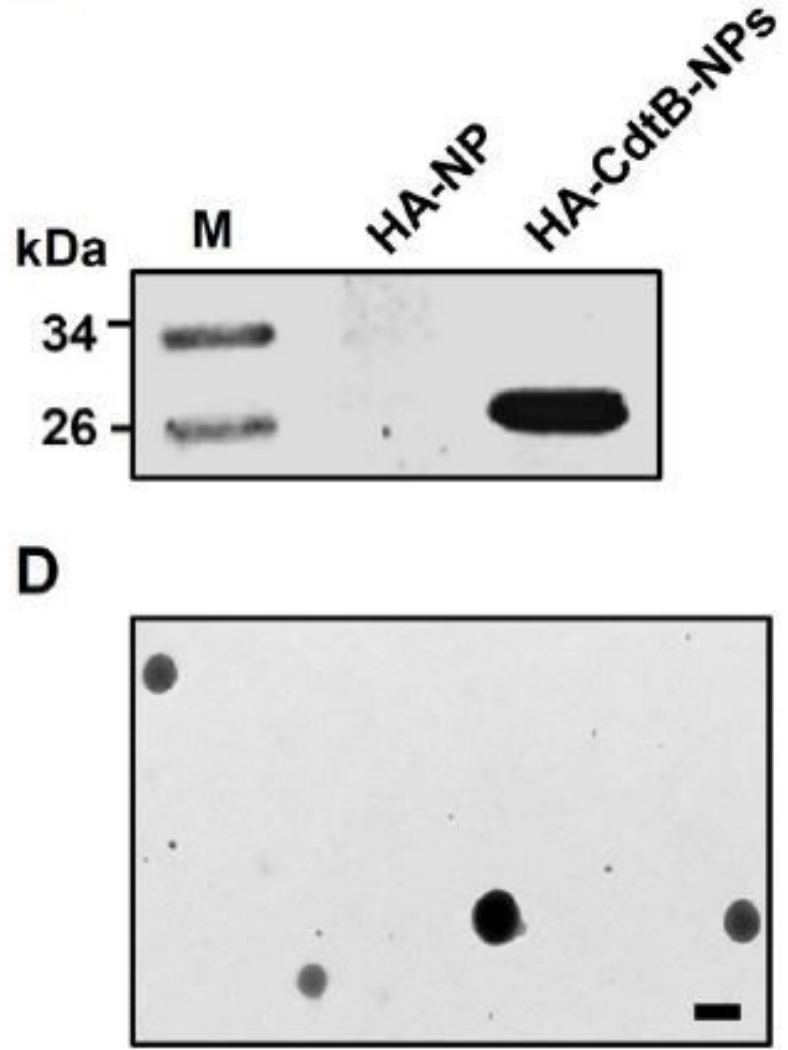

Figure 1

Characterization of HA-CdtB-NPs. SDS-PAGE analysis of (A) each recombinant CDT subunit and (B) HACdtB-NPs. Transmission electron micrographs of (C) HA-NPs and (D) HA-CdtB-NPs. Scale, $200 \mathrm{~nm}$. 
A

kDa M CdtA CdtB CdtC

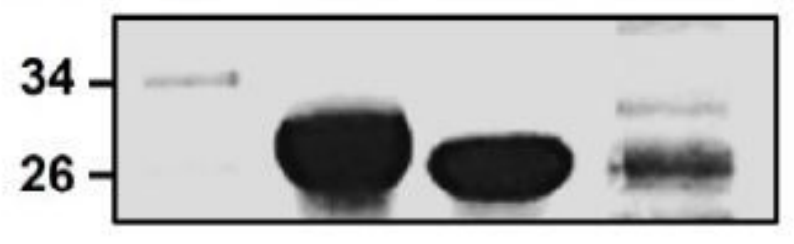

C

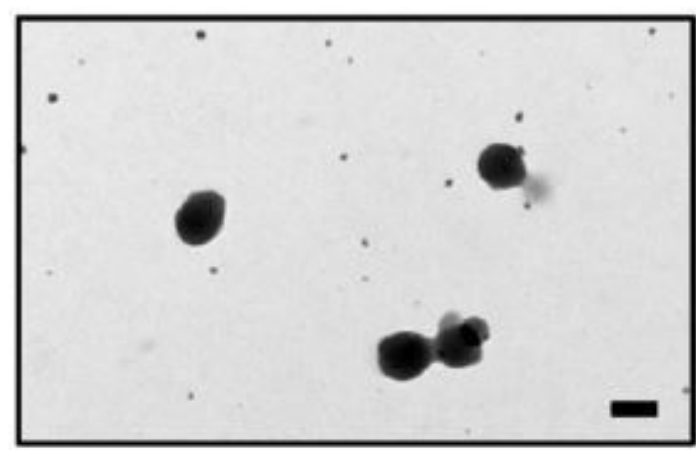

B
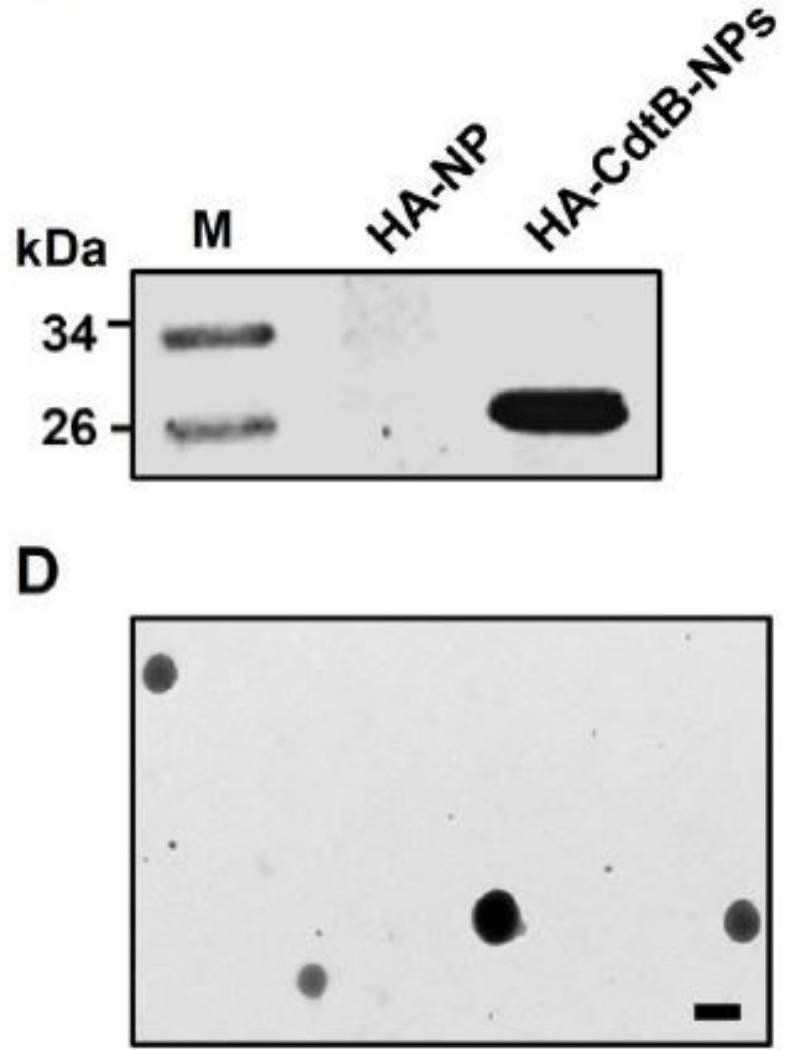

Figure 1

Characterization of HA-CdtB-NPs. SDS-PAGE analysis of (A) each recombinant CDT subunit and (B) HACdtB-NPs. Transmission electron micrographs of (C) HA-NPs and (D) HA-CdtB-NPs. Scale, $200 \mathrm{~nm}$. 
A B
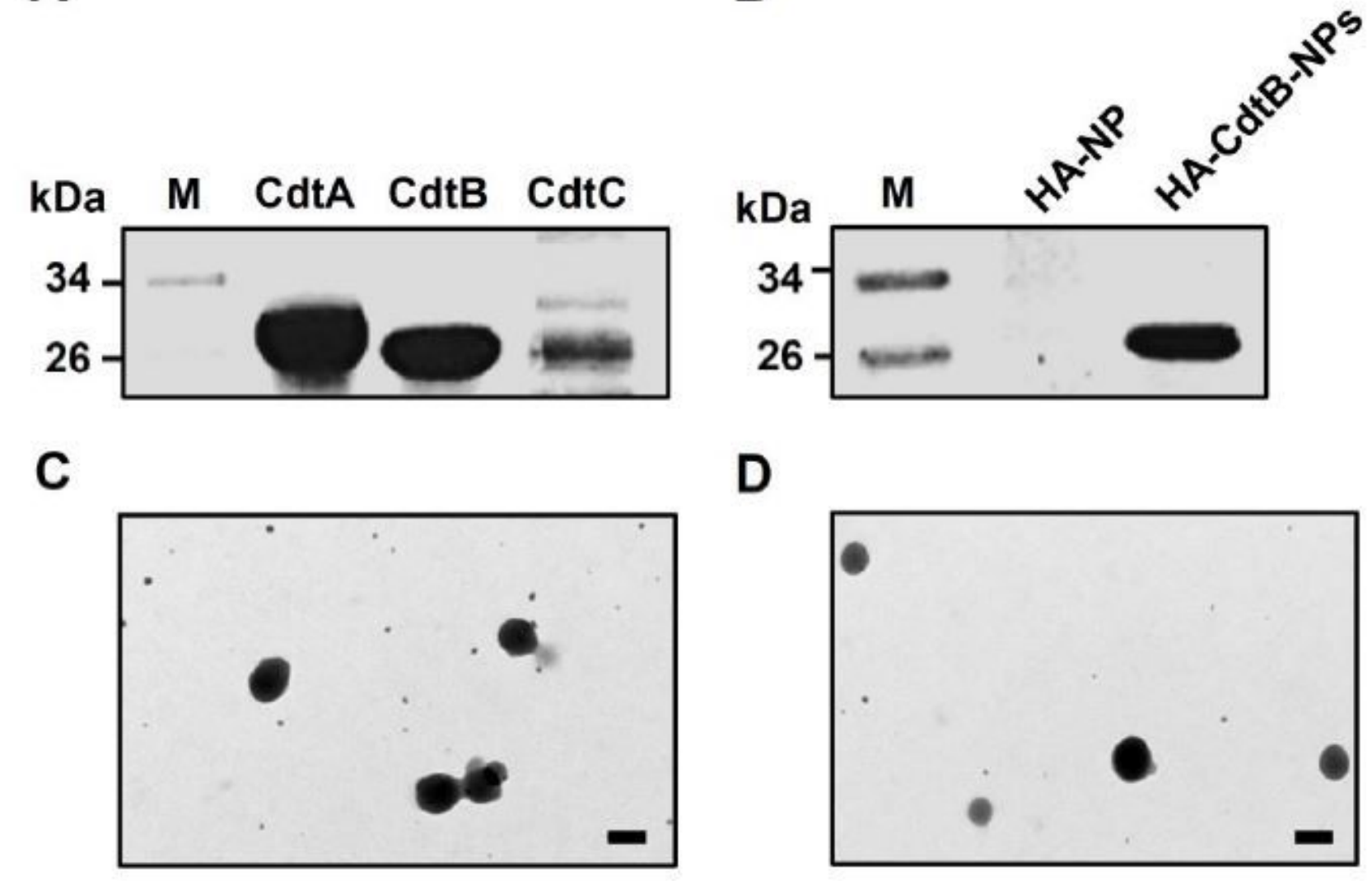

Figure 1

Characterization of HA-CdtB-NPs. SDS-PAGE analysis of (A) each recombinant CDT subunit and (B) HACdtB-NPs. Transmission electron micrographs of (C) HA-NPs and (D) HA-CdtB-NPs. Scale, $200 \mathrm{~nm}$. 
A

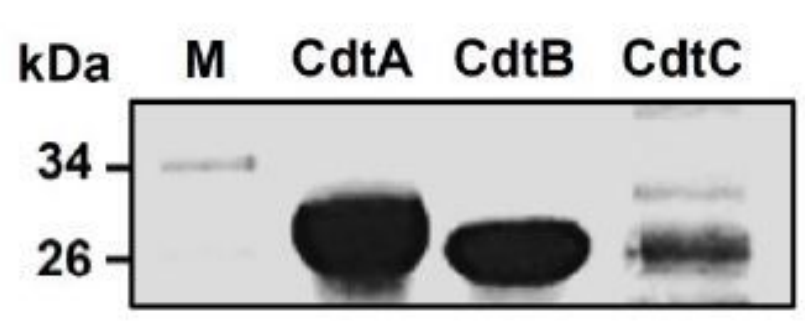

C

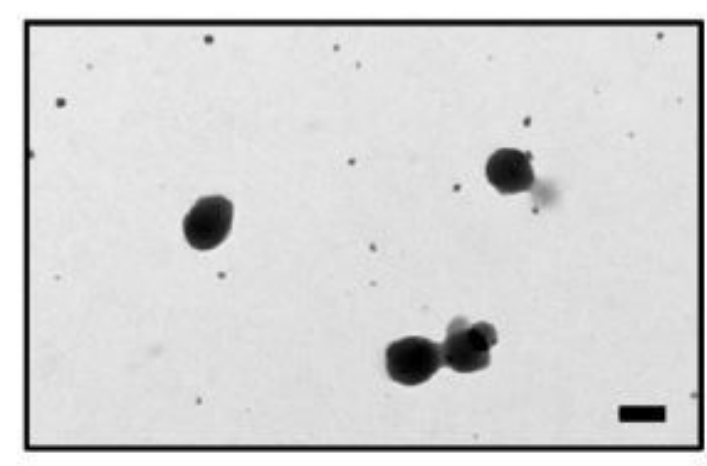

B
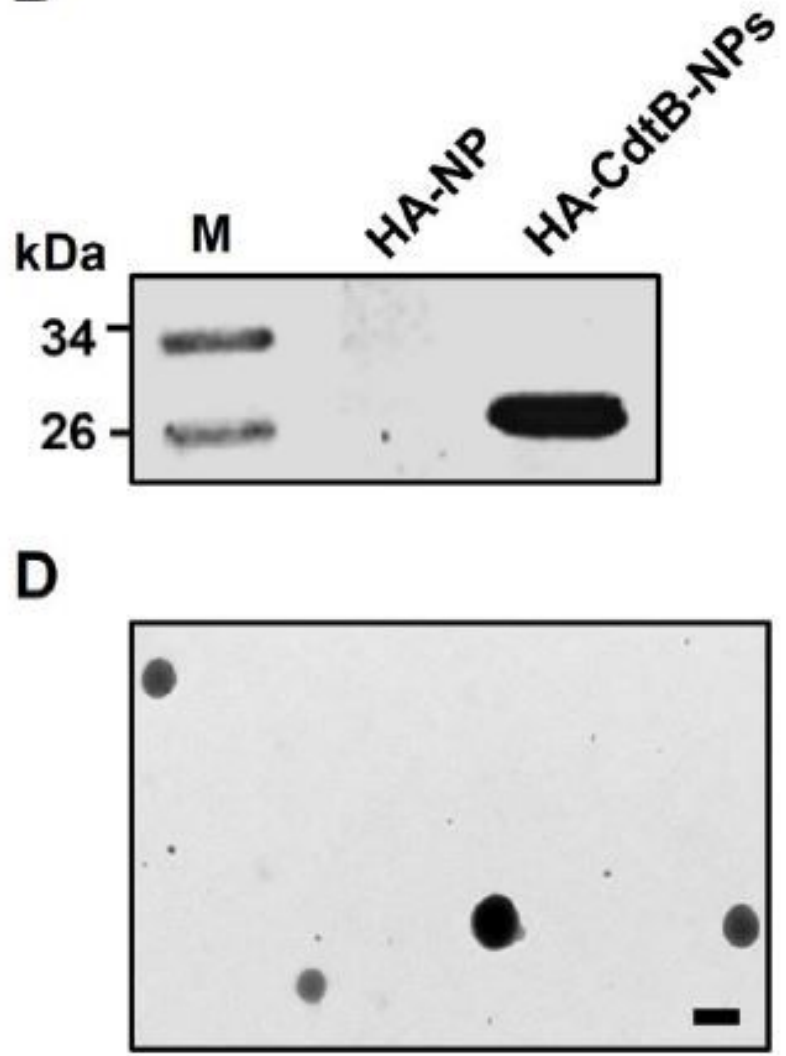

Figure 1

Characterization of HA-CdtB-NPs. SDS-PAGE analysis of (A) each recombinant CDT subunit and (B) HACdtB-NPs. Transmission electron micrographs of (C) HA-NPs and (D) HA-CdtB-NPs. Scale, $200 \mathrm{~nm}$. 


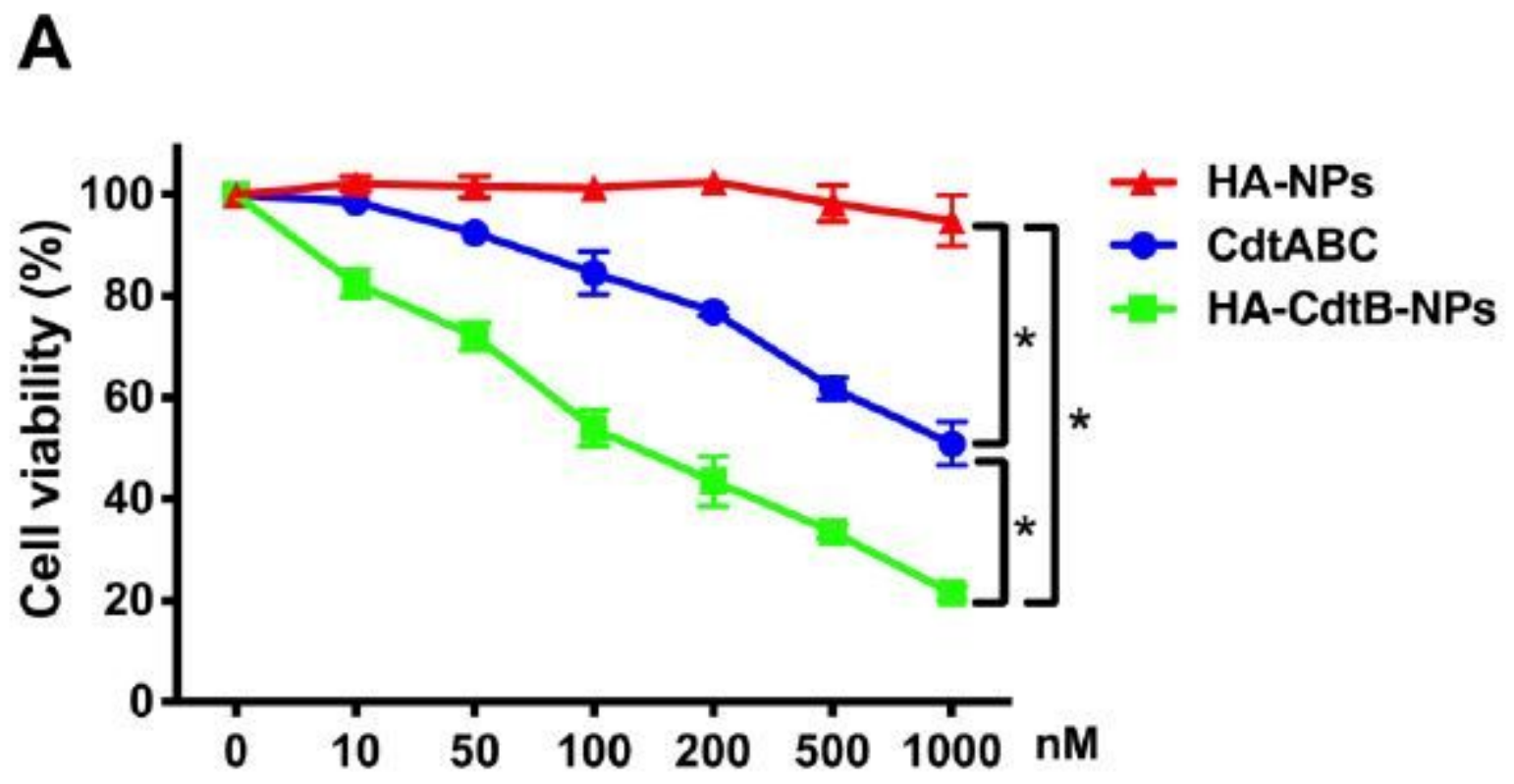

B

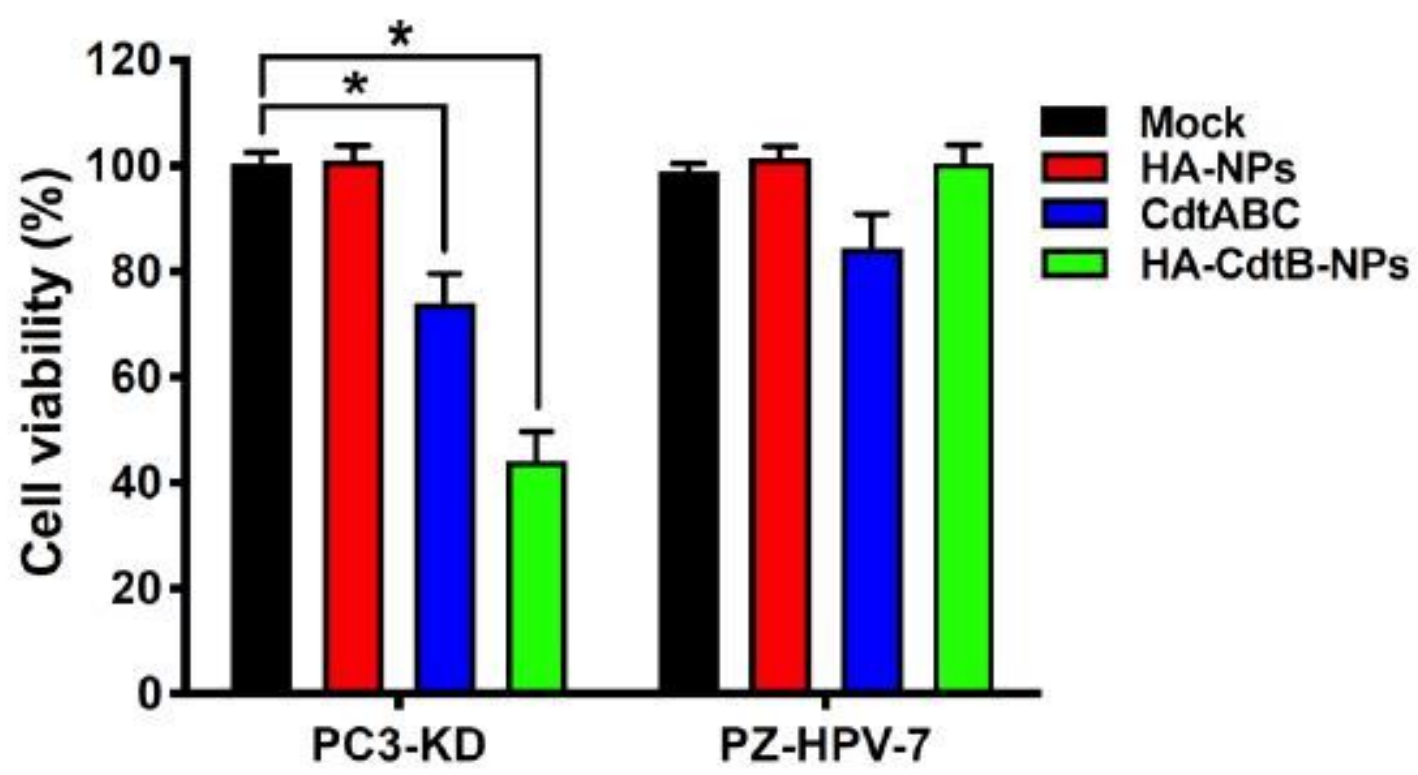

Figure 2

HA-CdtB-NPs inhibit PCa cell proliferation. (A) PC3-KD cells were treated with HA-NPs, CDT holotoxin or HA-CdtB-NPs at the indicated concentrations (0-1000 nM) for $48 \mathrm{~h}$. (B) PZ-HPV-7 and PC3-KD cells were treated with HA-NPs, CDT holotoxin or HA-CdtB-NPs at the concentration of $100 \mathrm{nM}$ for $48 \mathrm{~h}$. Cell viability was assessed using MTT assay. Statistical significance was evaluated using one-way ANOVA with post hoc test $(*, p<0.05)$. 


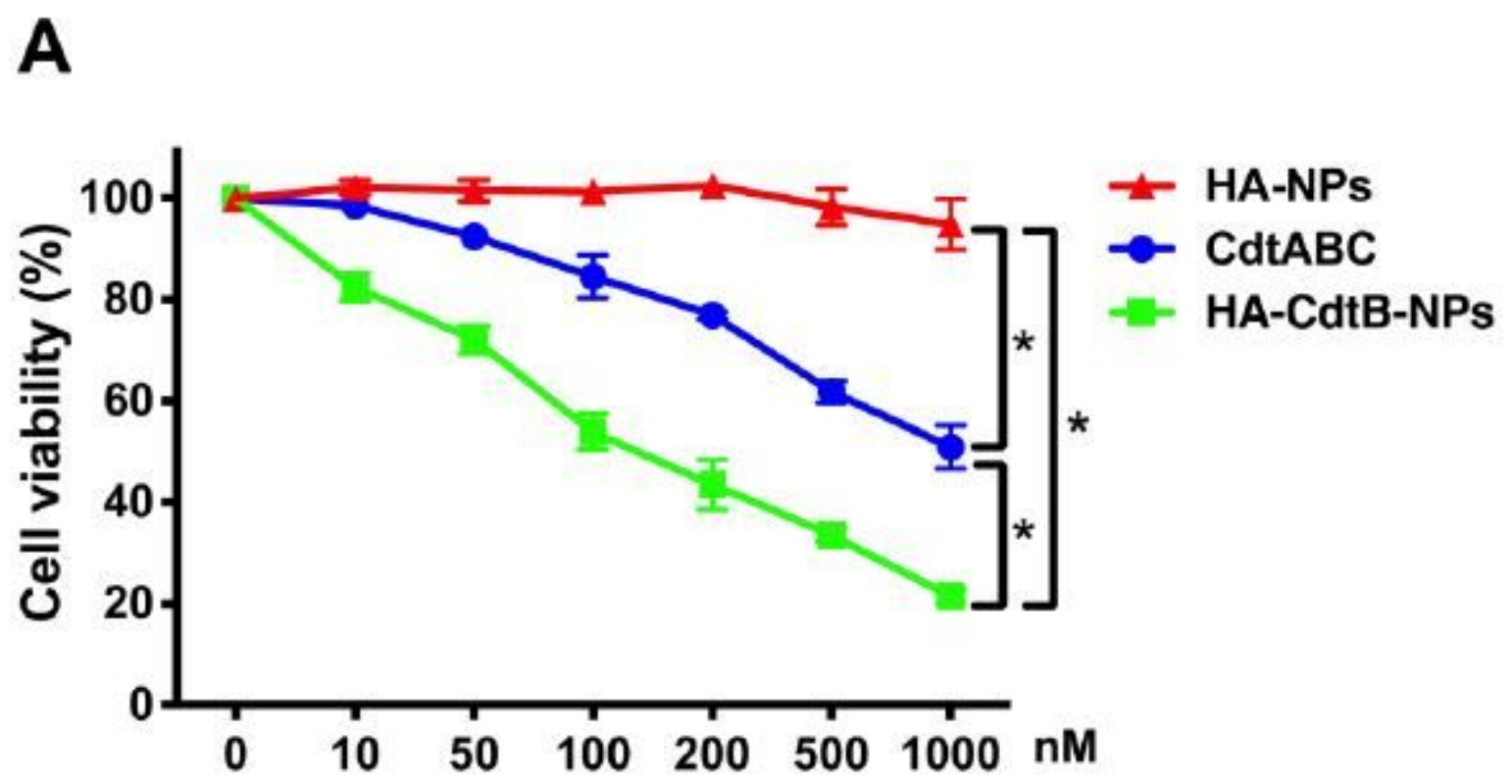

B

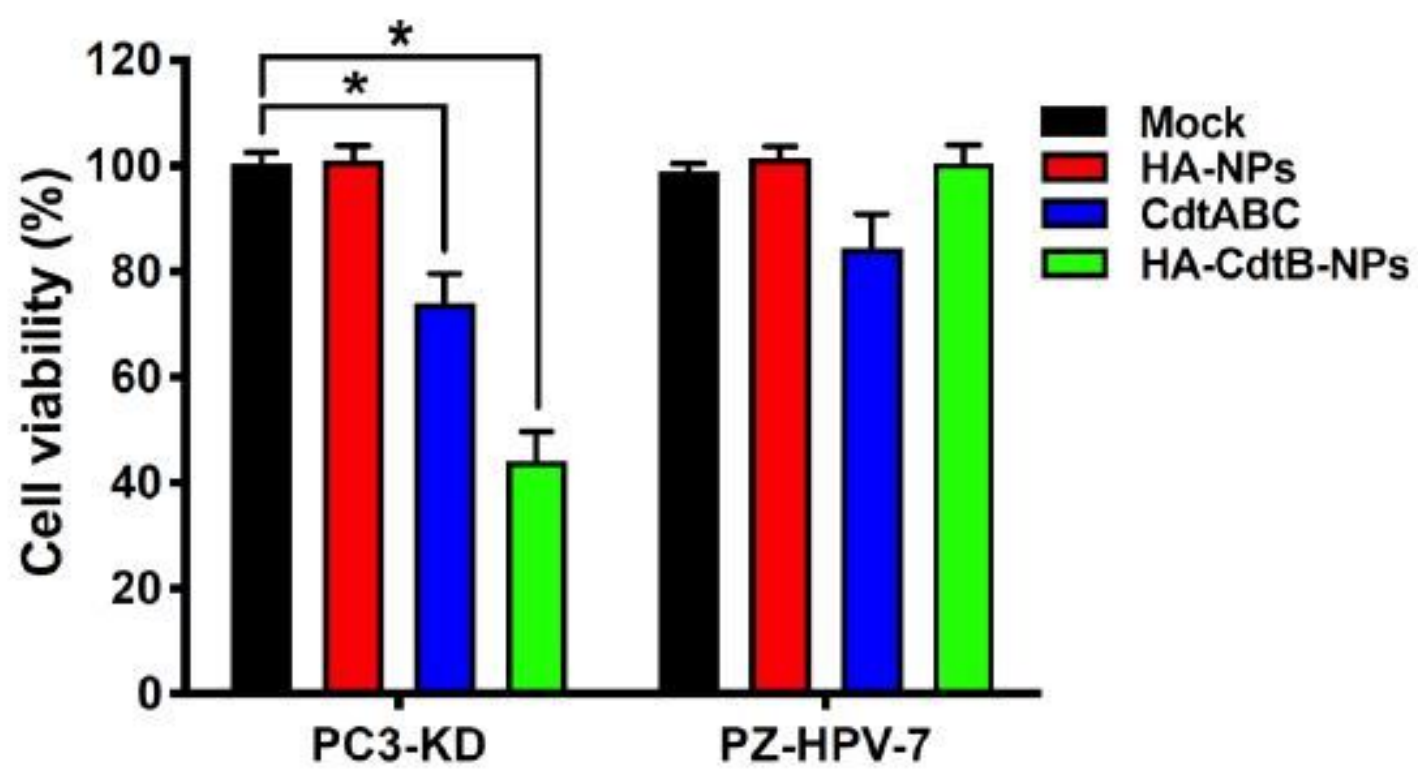

Figure 2

HA-CdtB-NPs inhibit PCa cell proliferation. (A) PC3-KD cells were treated with HA-NPs, CDT holotoxin or HA-CdtB-NPs at the indicated concentrations (0-1000 nM) for $48 \mathrm{~h}$. (B) PZ-HPV-7 and PC3-KD cells were treated with HA-NPs, CDT holotoxin or HA-CdtB-NPs at the concentration of $100 \mathrm{nM}$ for $48 \mathrm{~h}$. Cell viability was assessed using MTT assay. Statistical significance was evaluated using one-way ANOVA with post hoc test $(*, p<0.05)$. 


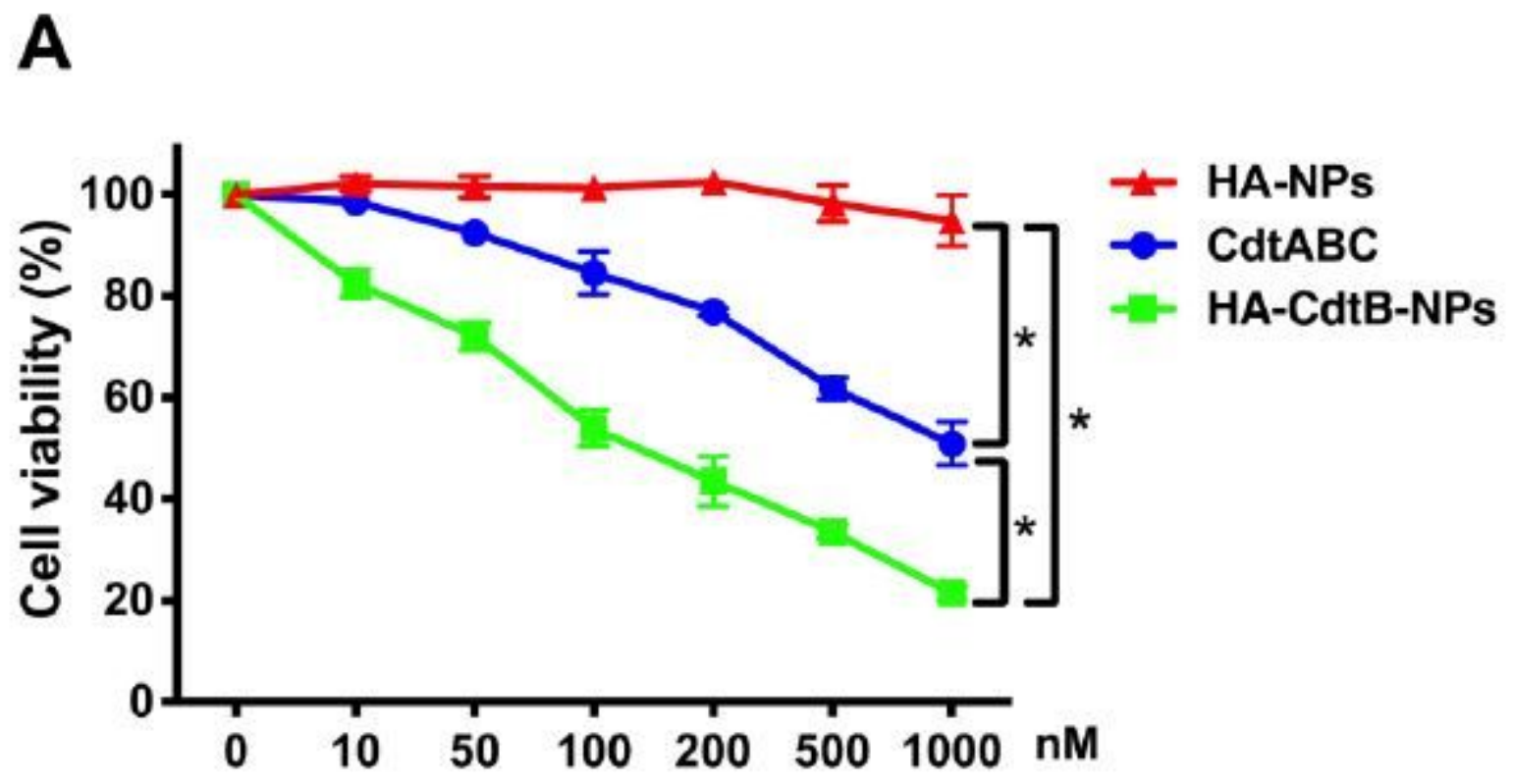

B

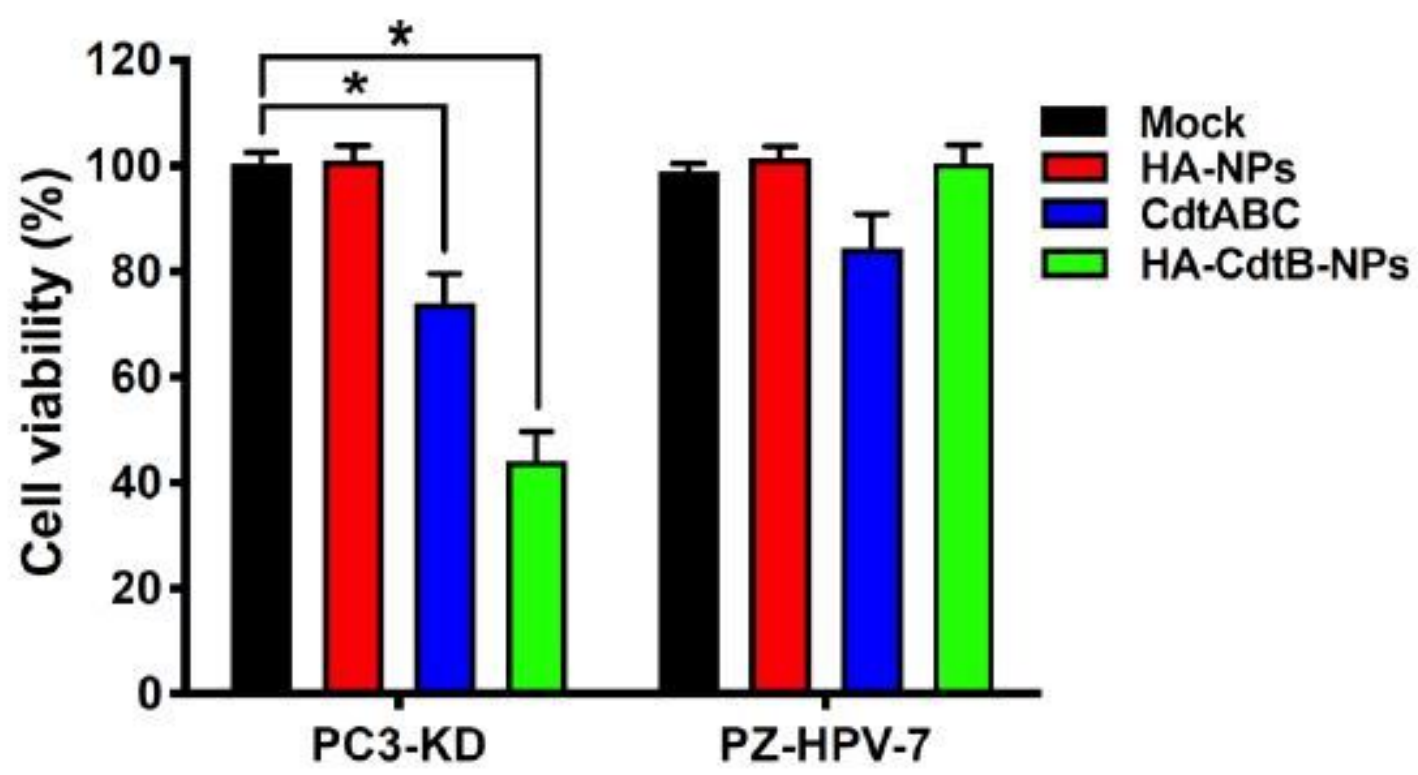

Figure 2

HA-CdtB-NPs inhibit PCa cell proliferation. (A) PC3-KD cells were treated with HA-NPs, CDT holotoxin or HA-CdtB-NPs at the indicated concentrations (0-1000 nM) for $48 \mathrm{~h}$. (B) PZ-HPV-7 and PC3-KD cells were treated with HA-NPs, CDT holotoxin or HA-CdtB-NPs at the concentration of $100 \mathrm{nM}$ for $48 \mathrm{~h}$. Cell viability was assessed using MTT assay. Statistical significance was evaluated using one-way ANOVA with post hoc test $(*, p<0.05)$. 


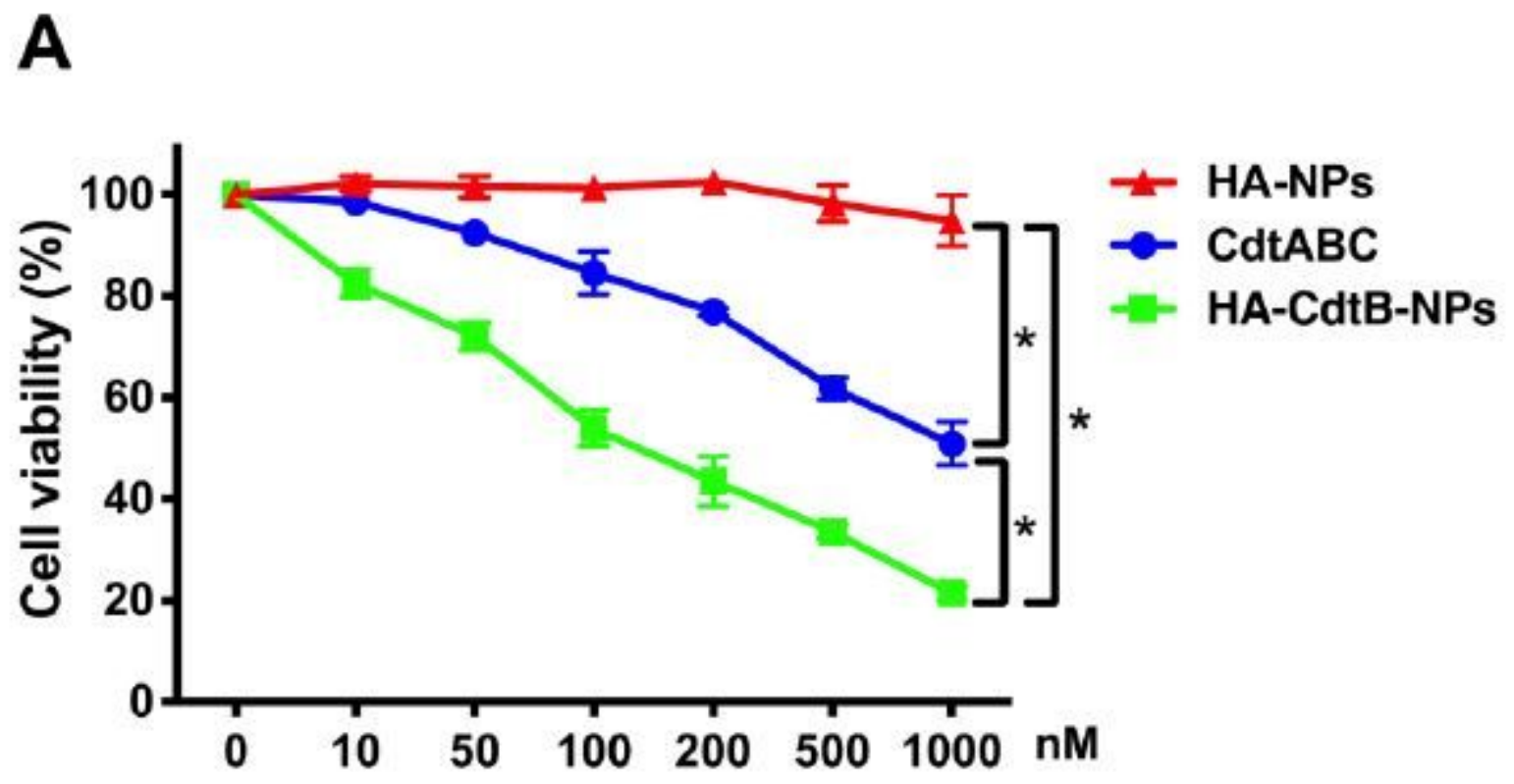

B

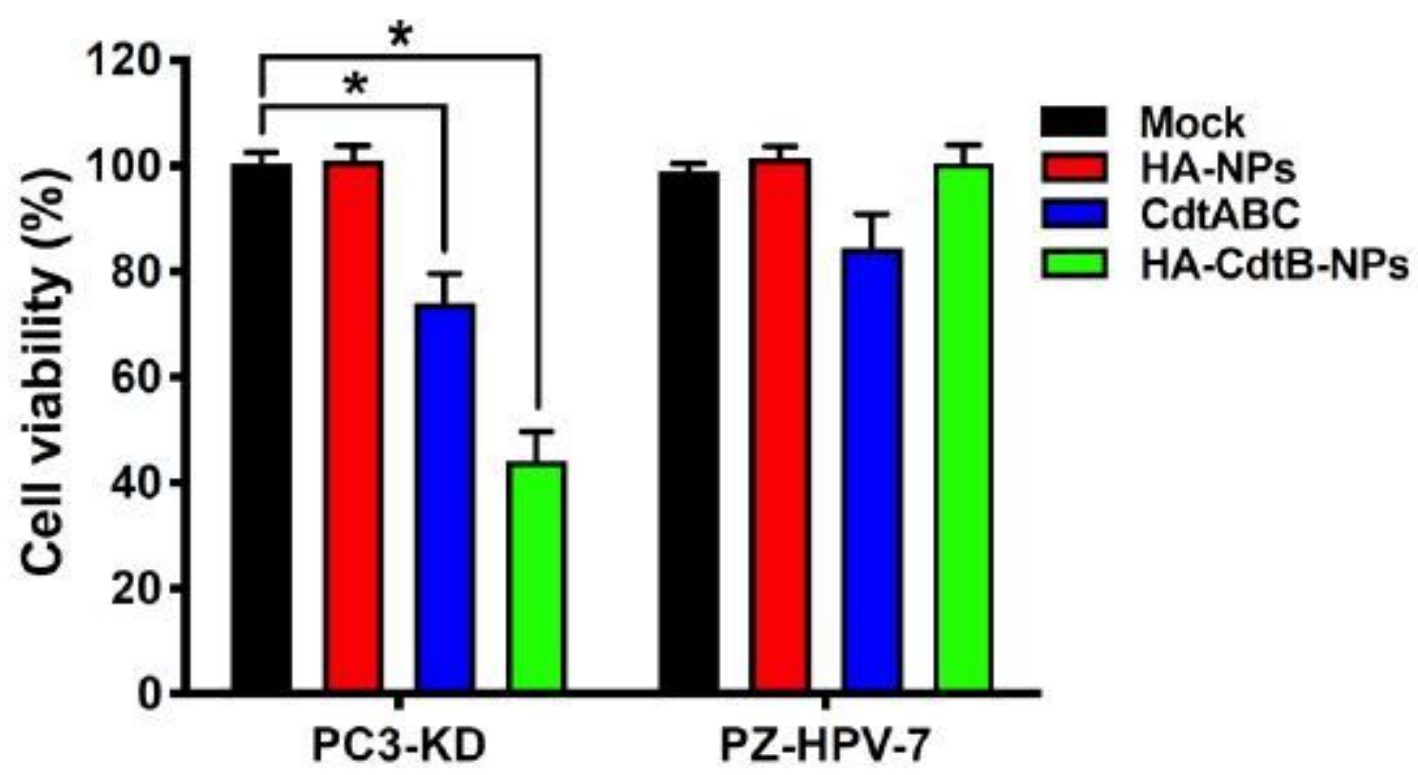

Figure 2

HA-CdtB-NPs inhibit PCa cell proliferation. (A) PC3-KD cells were treated with HA-NPs, CDT holotoxin or HA-CdtB-NPs at the indicated concentrations (0-1000 nM) for $48 \mathrm{~h}$. (B) PZ-HPV-7 and PC3-KD cells were treated with HA-NPs, CDT holotoxin or HA-CdtB-NPs at the concentration of $100 \mathrm{nM}$ for $48 \mathrm{~h}$. Cell viability was assessed using MTT assay. Statistical significance was evaluated using one-way ANOVA with post hoc test $(*, p<0.05)$. 


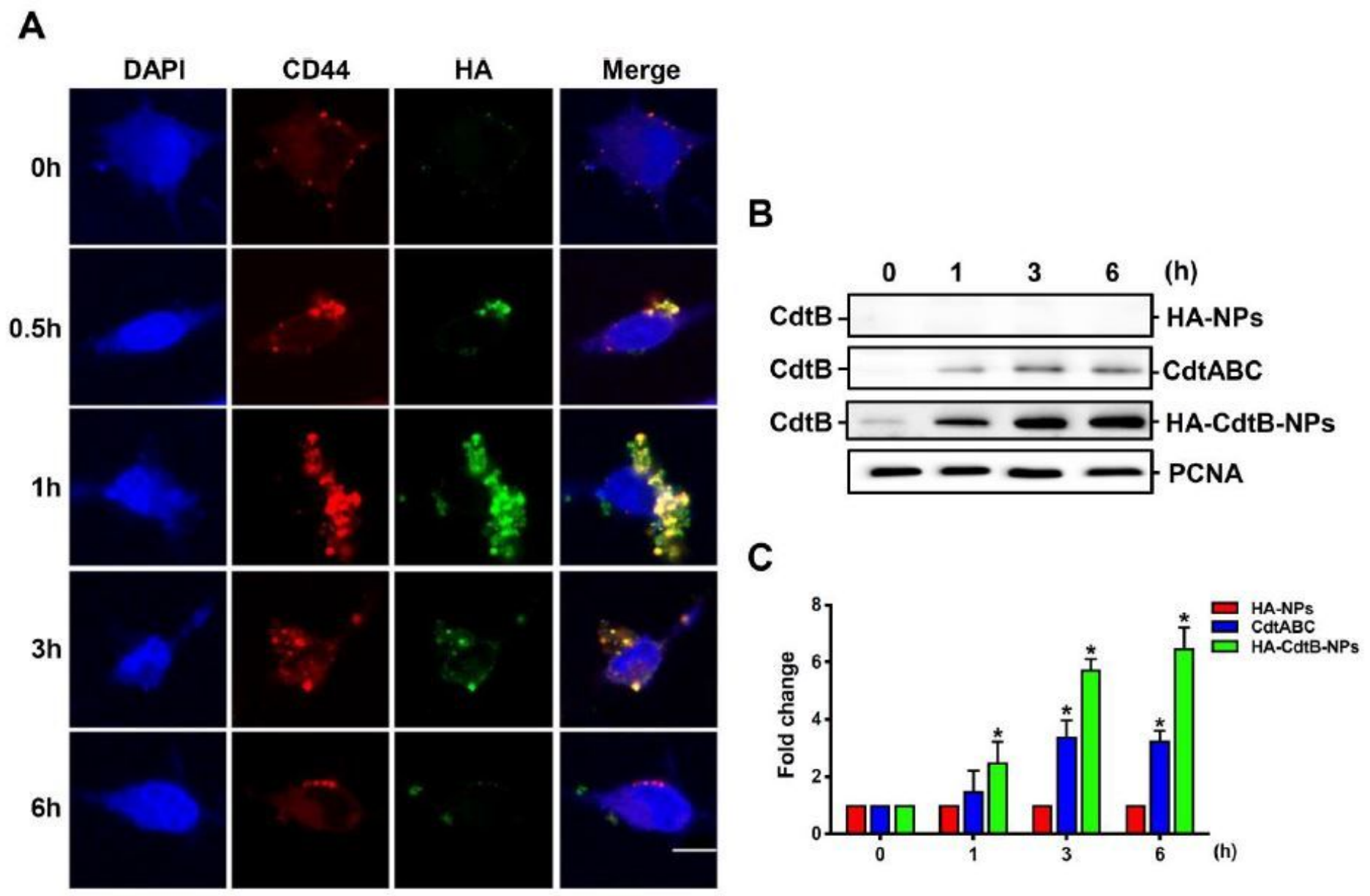

Figure 3

Delivery of HA-CdtB-NPs into PCa cells. (A) PC3-KD cells were treated with HA-CdtB-NPs-GFP for 0, 0.5, 1 , 3 , and $6 \mathrm{~h}$. The colocalization of CD44 and HA on the cell membrane was observed. Scale, $10 \mu \mathrm{m}$. (B) PC3-KD cells were exposed to $100 \mathrm{nM}$ HA-NPs, CDT holotoxin, and HA-CdtB-NPs, then incubated for the indicated times. The nuclear fraction from cell lysates was prepared and CdtB in the nucleus was analyzed by western blotting. Proliferating cell nuclear antigen (PCNA) was used as a loading control for nuclear protein. (C) The expression of CdtB in the nucleus was quantified for each time point. Statistical significance was evaluated using one-way ANOVA with post hoc test $(*, p<0.05)$. 


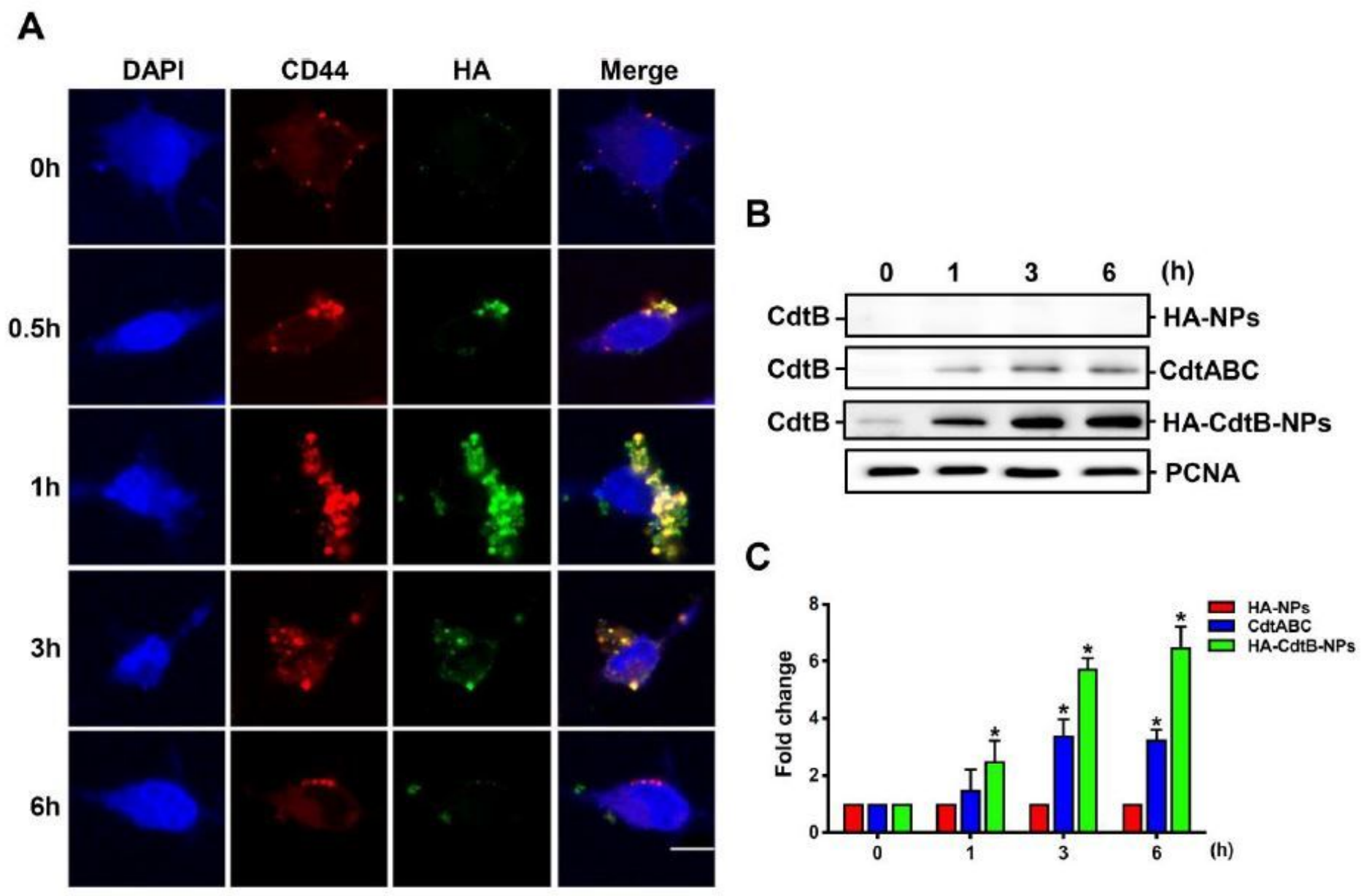

Figure 3

Delivery of HA-CdtB-NPs into PCa cells. (A) PC3-KD cells were treated with HA-CdtB-NPs-GFP for 0, 0.5, 1 , 3 , and $6 \mathrm{~h}$. The colocalization of CD44 and HA on the cell membrane was observed. Scale, $10 \mu \mathrm{m}$. (B) PC3-KD cells were exposed to $100 \mathrm{nM}$ HA-NPs, CDT holotoxin, and HA-CdtB-NPs, then incubated for the indicated times. The nuclear fraction from cell lysates was prepared and CdtB in the nucleus was analyzed by western blotting. Proliferating cell nuclear antigen (PCNA) was used as a loading control for nuclear protein. (C) The expression of CdtB in the nucleus was quantified for each time point. Statistical significance was evaluated using one-way ANOVA with post hoc test $(*, p<0.05)$. 


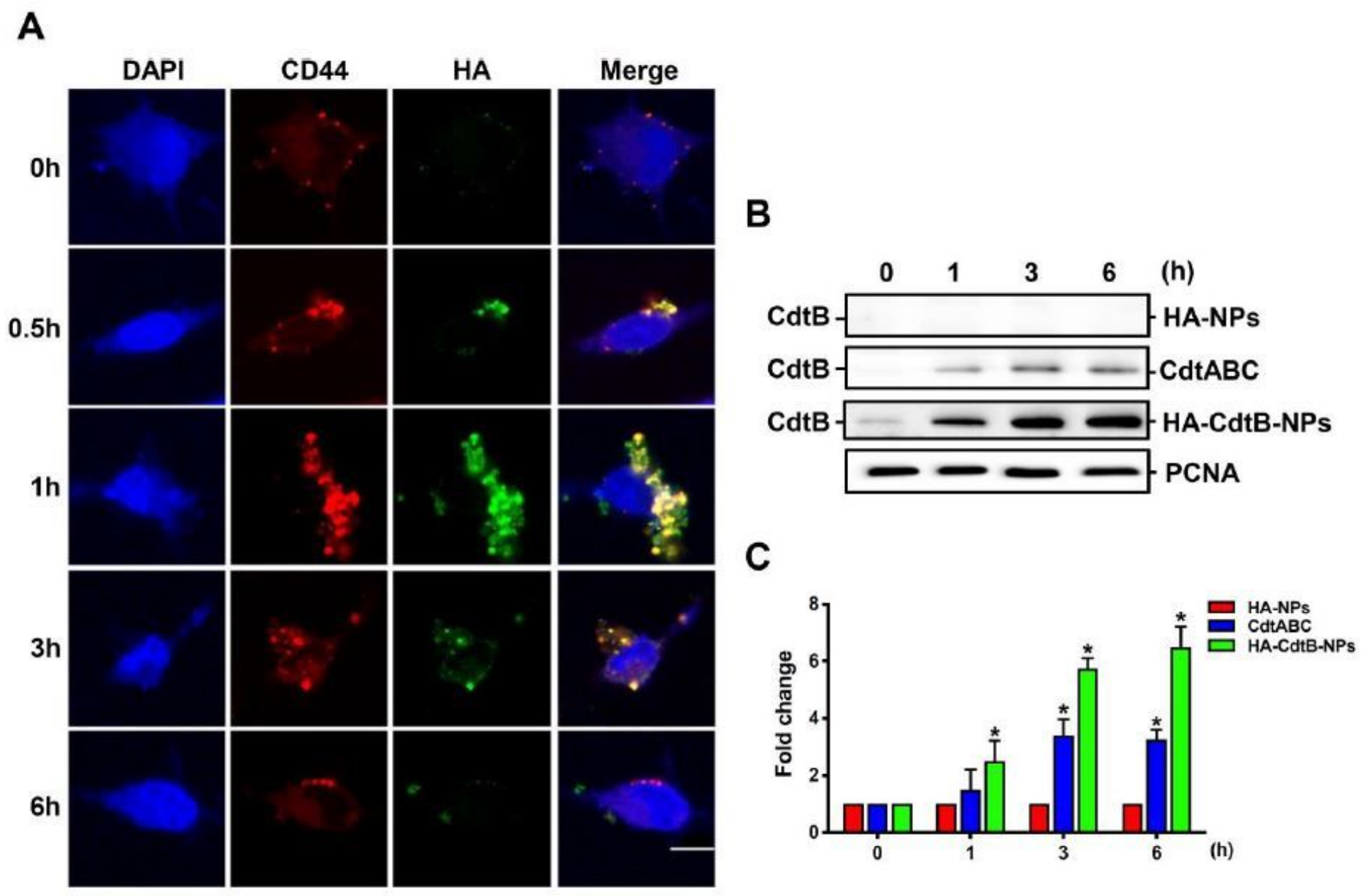

Figure 3

Delivery of HA-CdtB-NPs into PCa cells. (A) PC3-KD cells were treated with HA-CdtB-NPs-GFP for 0, 0.5, 1 , 3 , and $6 \mathrm{~h}$. The colocalization of CD44 and HA on the cell membrane was observed. Scale, $10 \mu \mathrm{m}$. (B) PC3-KD cells were exposed to $100 \mathrm{nM}$ HA-NPs, CDT holotoxin, and HA-CdtB-NPs, then incubated for the indicated times. The nuclear fraction from cell lysates was prepared and CdtB in the nucleus was analyzed by western blotting. Proliferating cell nuclear antigen (PCNA) was used as a loading control for nuclear protein. (C) The expression of CdtB in the nucleus was quantified for each time point. Statistical significance was evaluated using one-way ANOVA with post hoc test $(*, p<0.05)$. 


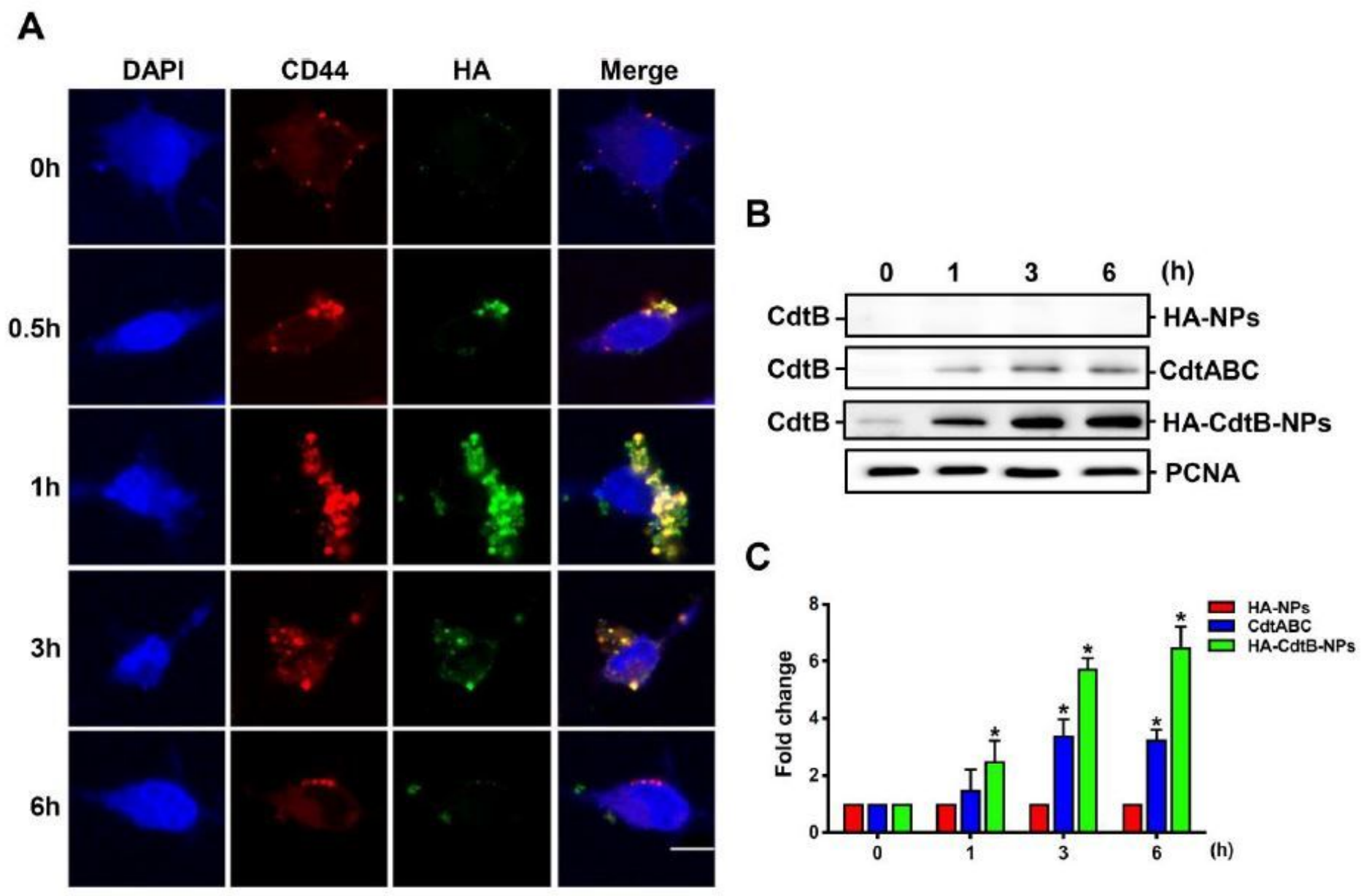

Figure 3

Delivery of HA-CdtB-NPs into PCa cells. (A) PC3-KD cells were treated with HA-CdtB-NPs-GFP for 0, 0.5, 1 , 3 , and $6 \mathrm{~h}$. The colocalization of CD44 and HA on the cell membrane was observed. Scale, $10 \mu \mathrm{m}$. (B) PC3-KD cells were exposed to $100 \mathrm{nM}$ HA-NPs, CDT holotoxin, and HA-CdtB-NPs, then incubated for the indicated times. The nuclear fraction from cell lysates was prepared and CdtB in the nucleus was analyzed by western blotting. Proliferating cell nuclear antigen (PCNA) was used as a loading control for nuclear protein. (C) The expression of CdtB in the nucleus was quantified for each time point. Statistical significance was evaluated using one-way ANOVA with post hoc test $(*, p<0.05)$. 
A

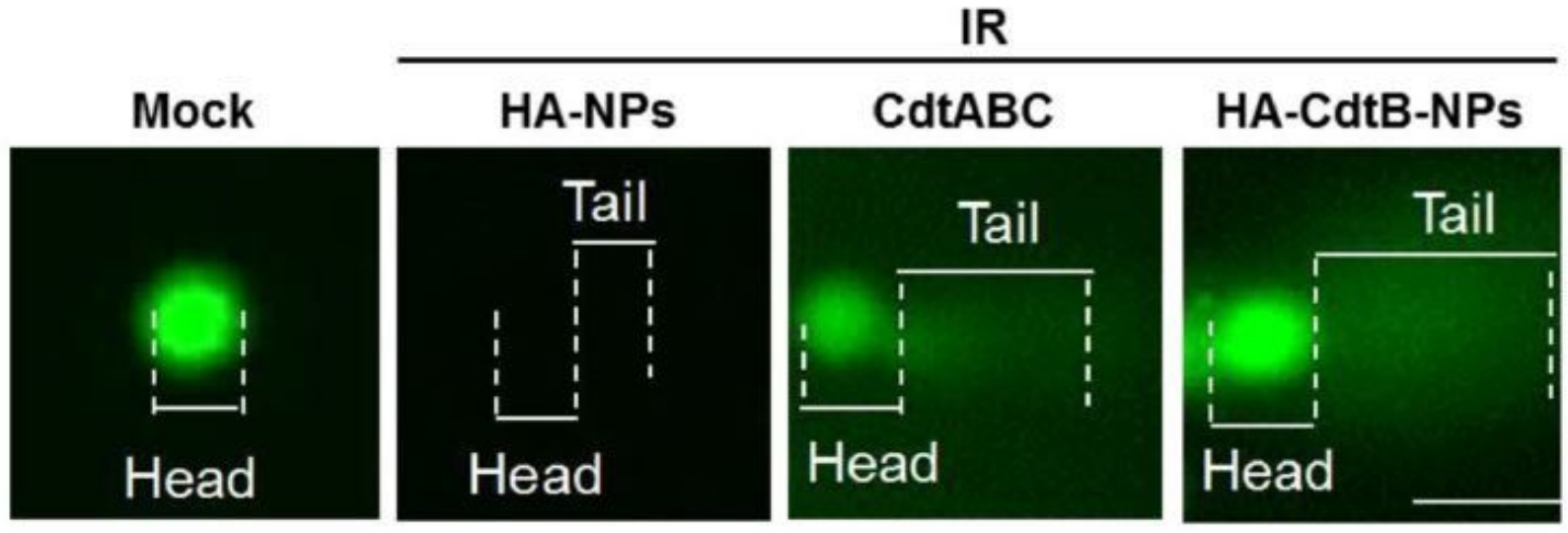

B

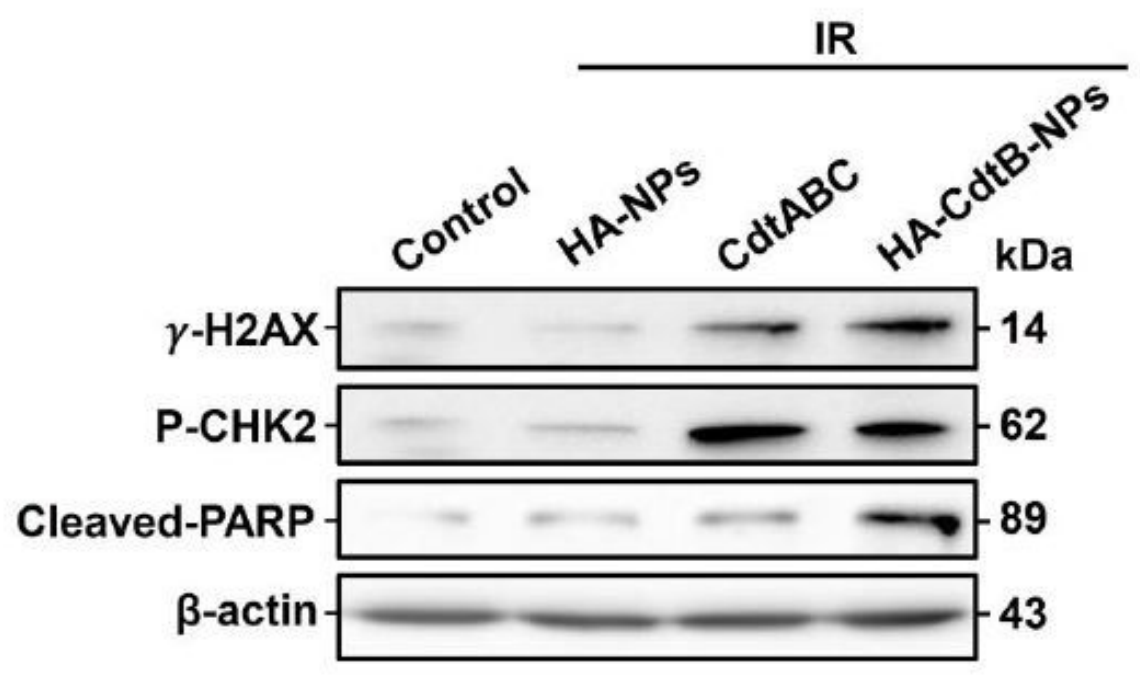

Figure 4

HA-CdtB-NPs enhance IR-induced DSB in PCa cells. PC3-KD cells were mock-treated or exposed to IR (2 Gy) followed by incubation with 100 HA-NPs, CDT holotoxin, and HA-CdtB-NPs. (A) Visualization of the comet tail showed IR-induced DSB in PC3-KD cells after $24 \mathrm{~h}$ incubation. Scale, $10 \mu \mathrm{m}$. (B) Western blot analysis for DNA damage-related proteins, $\mathrm{\gamma}-\mathrm{H} 2 \mathrm{AX}, \mathrm{p}-\mathrm{CHK} 2$, and cleaved PARP after $48 \mathrm{~h}$ incubation. $\beta$ actin was used as a loading control. 
A

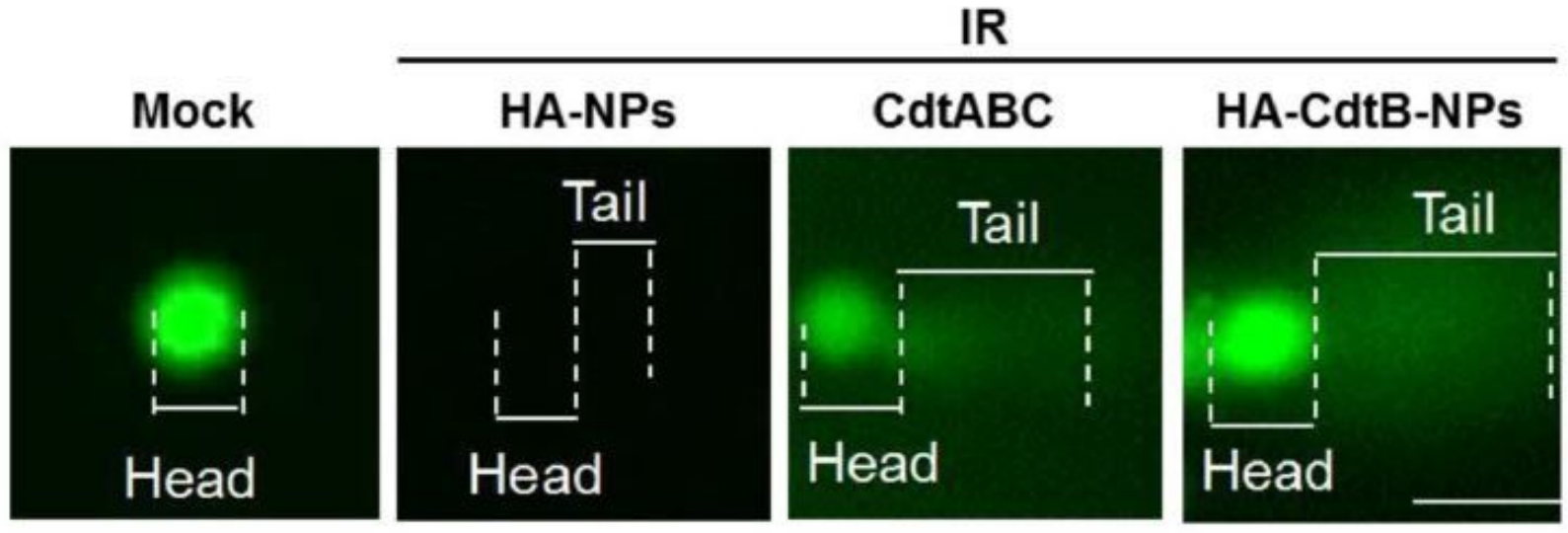

B

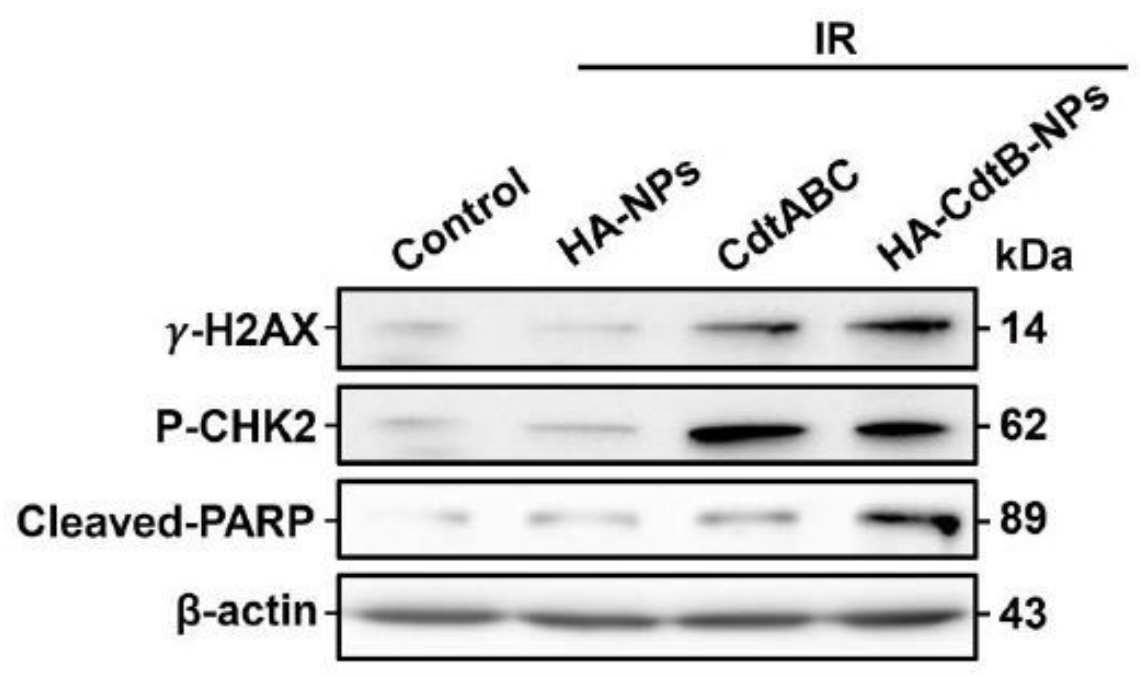

Figure 4

HA-CdtB-NPs enhance IR-induced DSB in PCa cells. PC3-KD cells were mock-treated or exposed to IR (2 Gy) followed by incubation with 100 HA-NPs, CDT holotoxin, and HA-CdtB-NPs. (A) Visualization of the comet tail showed IR-induced DSB in PC3-KD cells after $24 \mathrm{~h}$ incubation. Scale, $10 \mu \mathrm{m}$. (B) Western blot analysis for DNA damage-related proteins, $\mathrm{\gamma}-\mathrm{H} 2 \mathrm{AX}, \mathrm{p}-\mathrm{CHK} 2$, and cleaved PARP after $48 \mathrm{~h}$ incubation. $\beta$ actin was used as a loading control. 
A

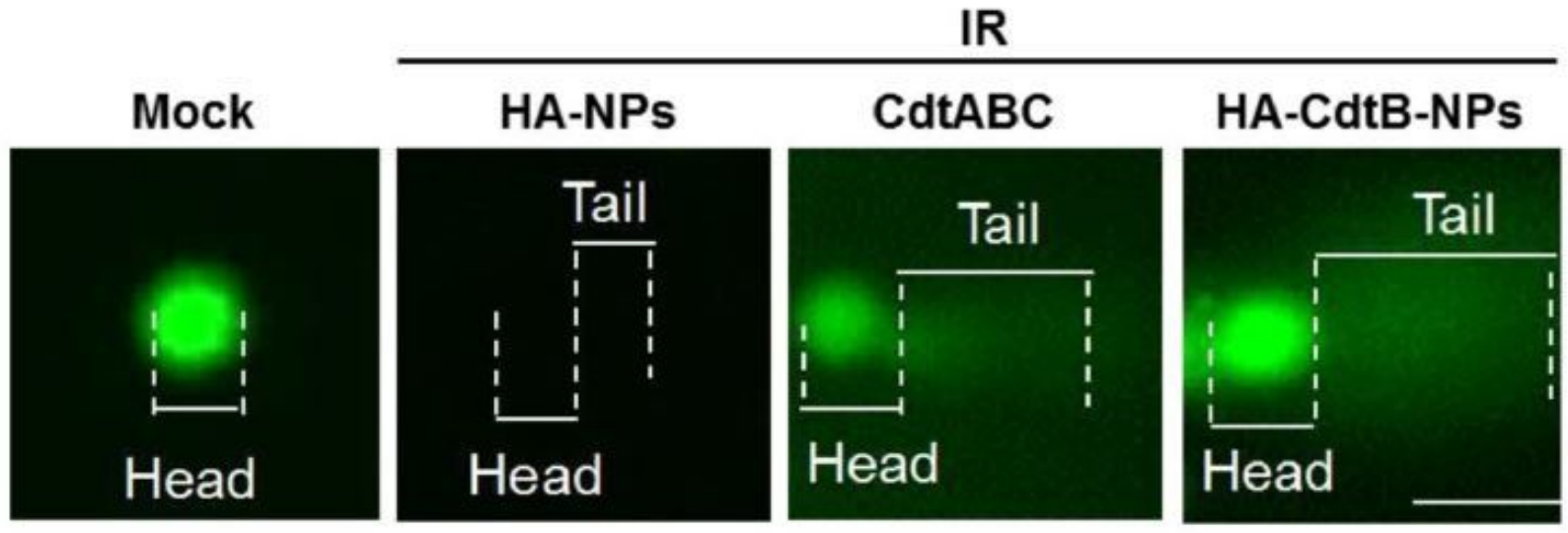

B

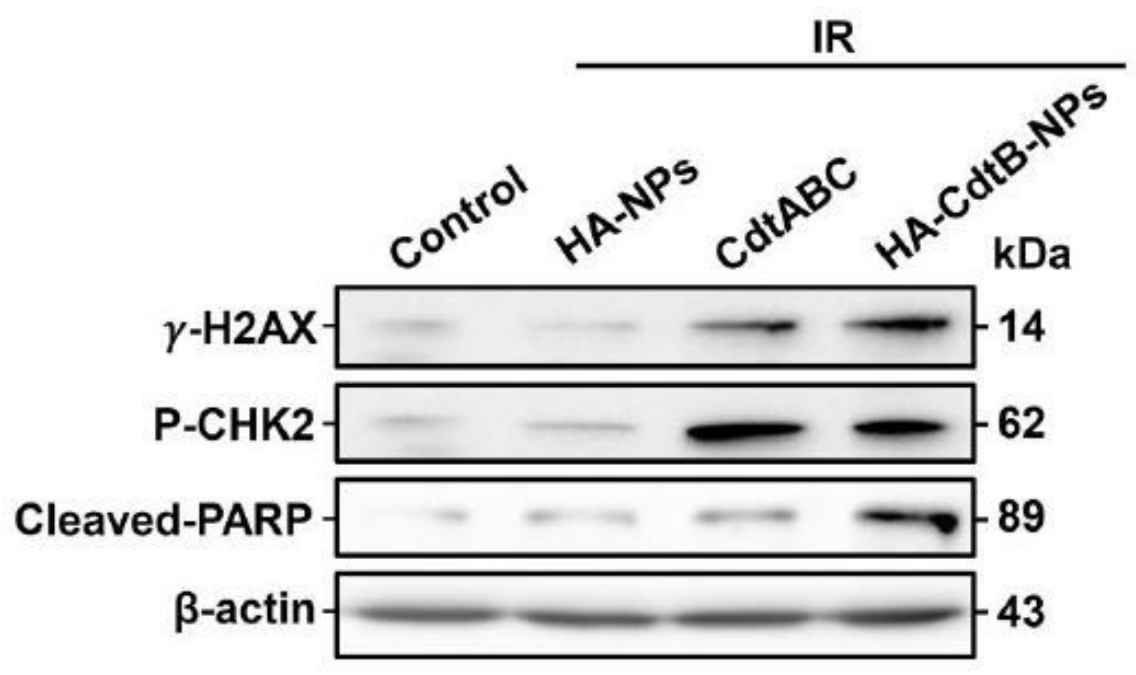

Figure 4

HA-CdtB-NPs enhance IR-induced DSB in PCa cells. PC3-KD cells were mock-treated or exposed to IR (2 Gy) followed by incubation with 100 HA-NPs, CDT holotoxin, and HA-CdtB-NPs. (A) Visualization of the comet tail showed IR-induced DSB in PC3-KD cells after $24 \mathrm{~h}$ incubation. Scale, $10 \mu \mathrm{m}$. (B) Western blot analysis for DNA damage-related proteins, $\mathrm{\gamma}-\mathrm{H} 2 \mathrm{AX}, \mathrm{p}-\mathrm{CHK} 2$, and cleaved PARP after $48 \mathrm{~h}$ incubation. $\beta$ actin was used as a loading control. 
A

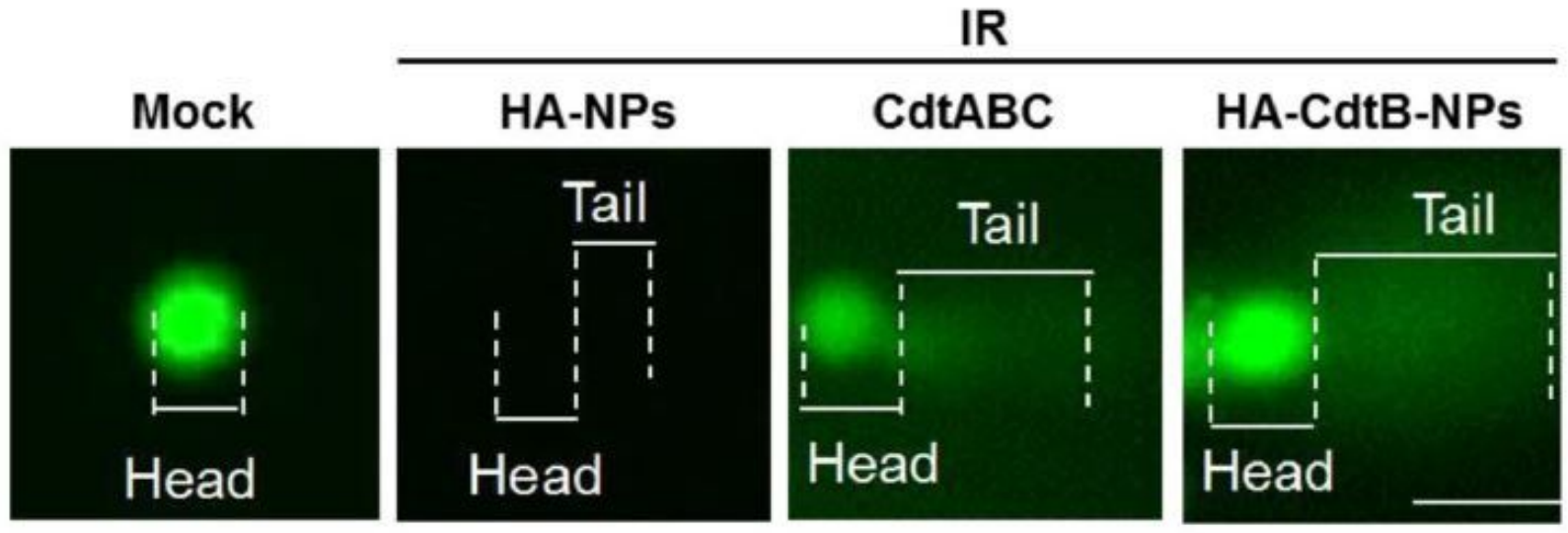

B

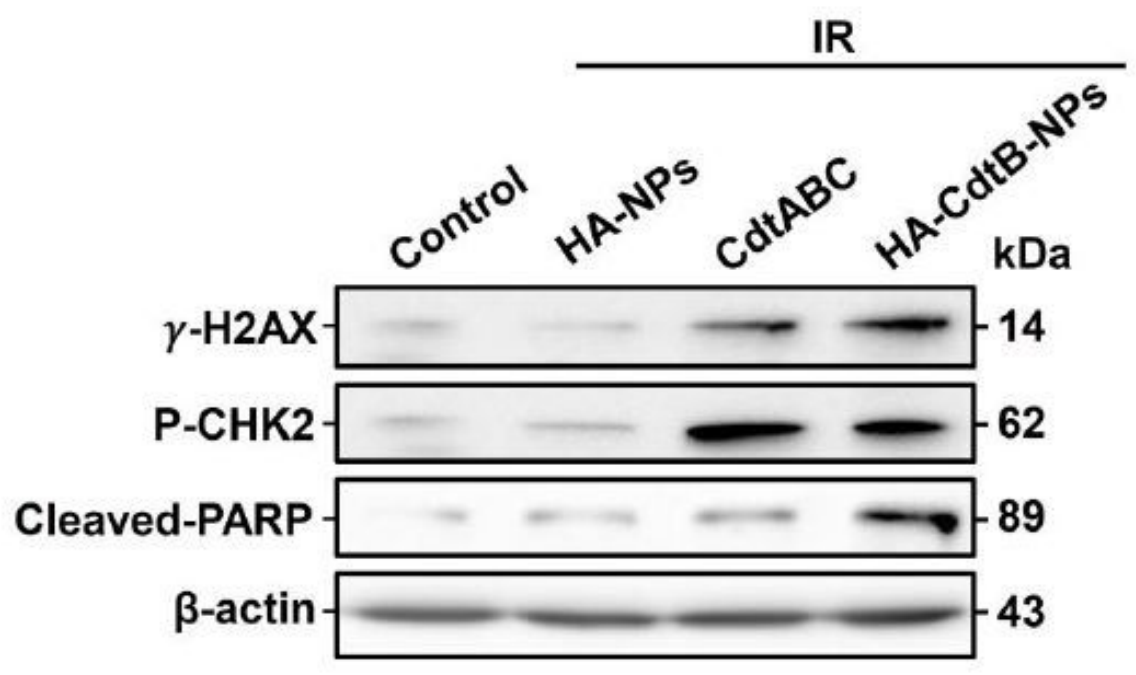

Figure 4

HA-CdtB-NPs enhance IR-induced DSB in PCa cells. PC3-KD cells were mock-treated or exposed to IR (2 Gy) followed by incubation with 100 HA-NPs, CDT holotoxin, and HA-CdtB-NPs. (A) Visualization of the comet tail showed IR-induced DSB in PC3-KD cells after $24 \mathrm{~h}$ incubation. Scale, $10 \mu \mathrm{m}$. (B) Western blot analysis for DNA damage-related proteins, $\mathrm{\gamma}-\mathrm{H} 2 \mathrm{AX}, \mathrm{p}-\mathrm{CHK} 2$, and cleaved PARP after $48 \mathrm{~h}$ incubation. $\beta$ actin was used as a loading control. 
A

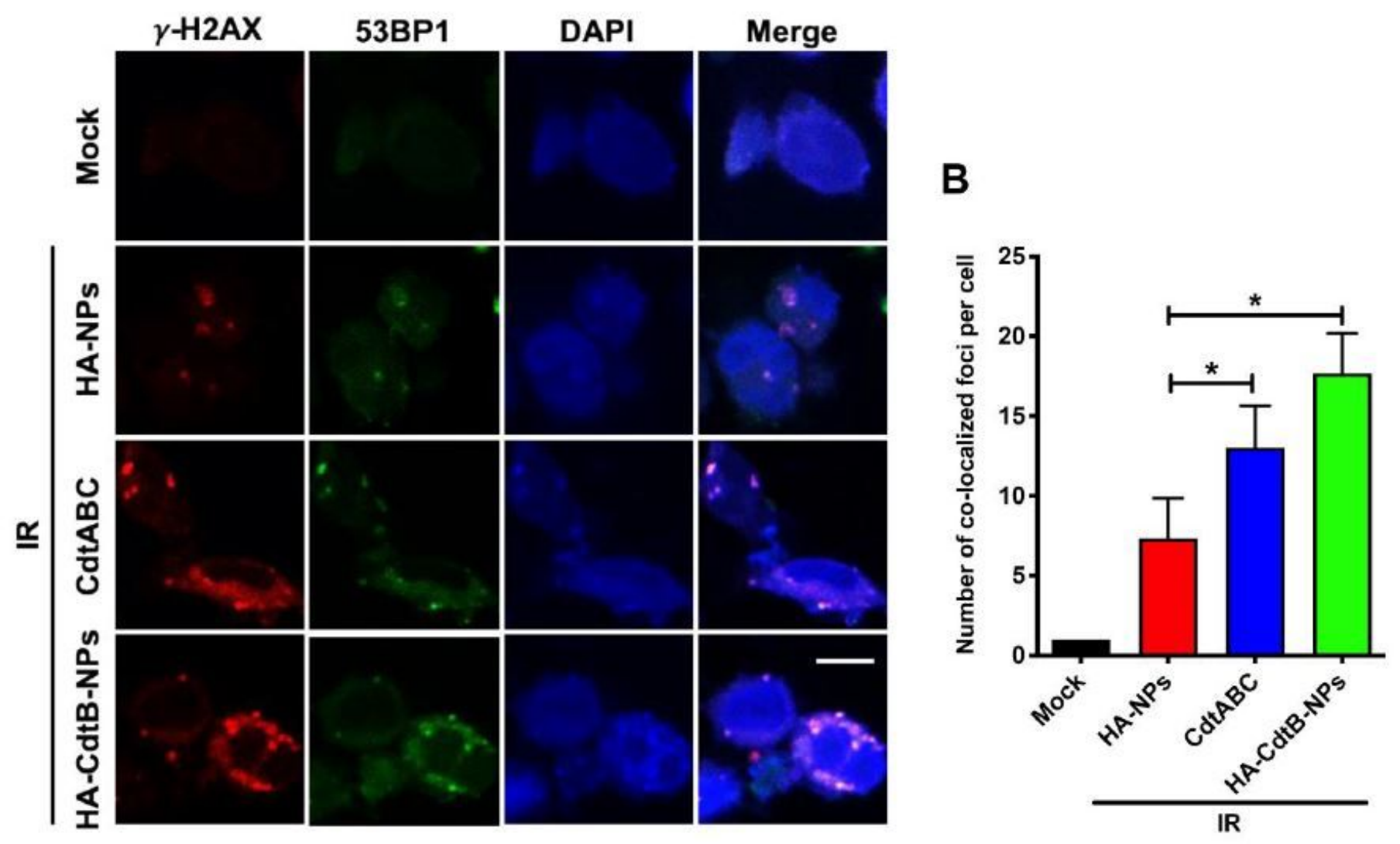

Figure 5

HA-CdtB-NPs sensitize PCa cells to radiation by promoting DSB. PC3-KD cells were mock-treated or exposed to IR (2 Gy) then incubated with $100 \mathrm{nM}$ HA-NPs, CDT holotoxin, and HA-CdtB-NPs for $24 \mathrm{~h}$. (A) Fluorescent immunostaining of $\mathrm{Y}-\mathrm{H} 2 \mathrm{AX}$ (red) and 53BP1 (green) was shown. DAPI (blue) was used as a tracer for cell nucleus. Scale, $10 \mu \mathrm{m}$. (B) The foci of $\mathrm{y}-\mathrm{H} 2 \mathrm{AX}$ and 53BP1 colocalization in the nuclei were counted. Statistical significance was evaluated using one-way ANOVA with post hoc test $(*, p<0.05)$. 
A

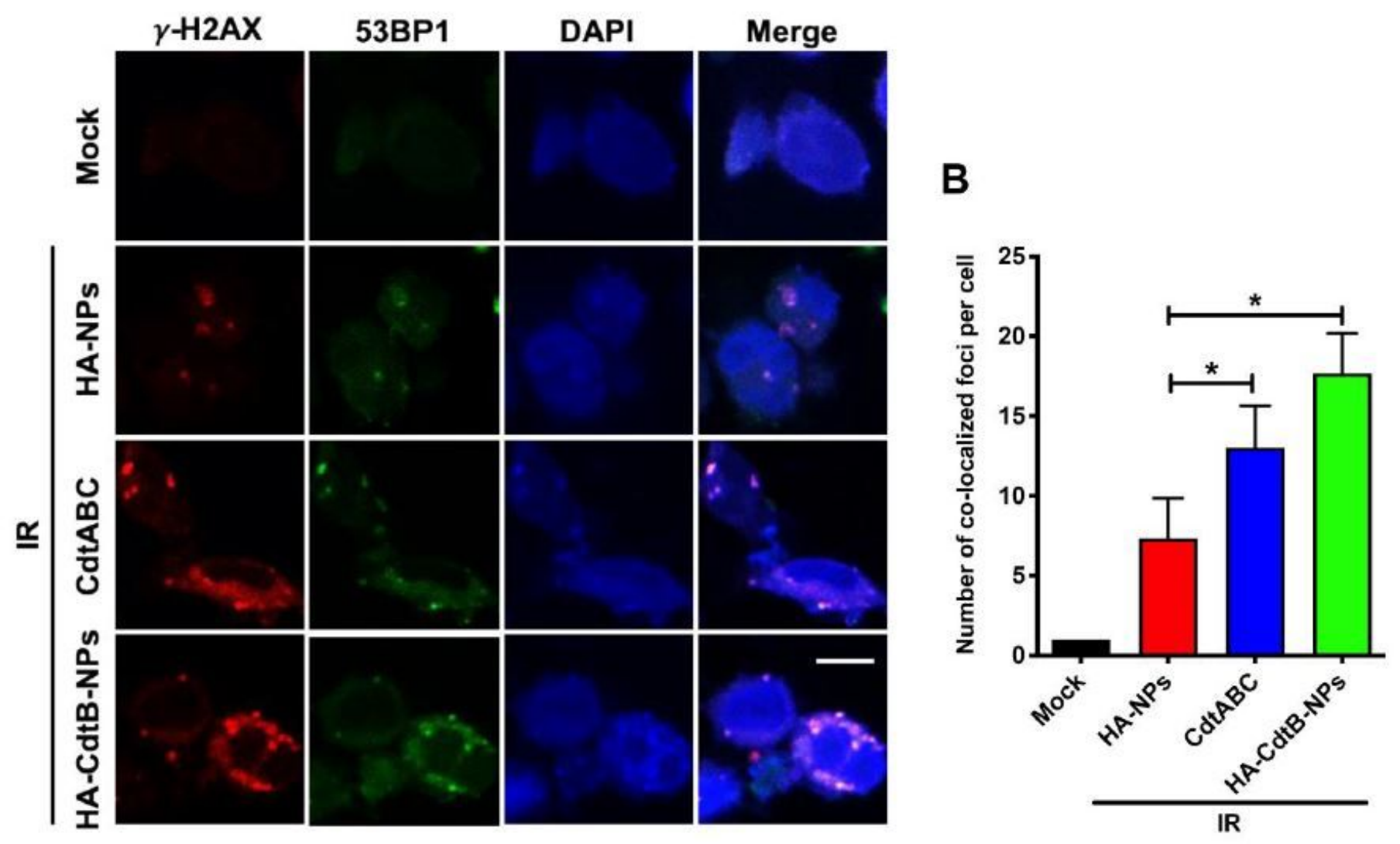

Figure 5

HA-CdtB-NPs sensitize PCa cells to radiation by promoting DSB. PC3-KD cells were mock-treated or exposed to IR (2 Gy) then incubated with $100 \mathrm{nM}$ HA-NPs, CDT holotoxin, and HA-CdtB-NPs for $24 \mathrm{~h}$. (A) Fluorescent immunostaining of $\mathrm{Y}-\mathrm{H} 2 \mathrm{AX}$ (red) and 53BP1 (green) was shown. DAPI (blue) was used as a tracer for cell nucleus. Scale, $10 \mu \mathrm{m}$. (B) The foci of $\mathrm{y}-\mathrm{H} 2 \mathrm{AX}$ and 53BP1 colocalization in the nuclei were counted. Statistical significance was evaluated using one-way ANOVA with post hoc test $(*, p<0.05)$. 
A

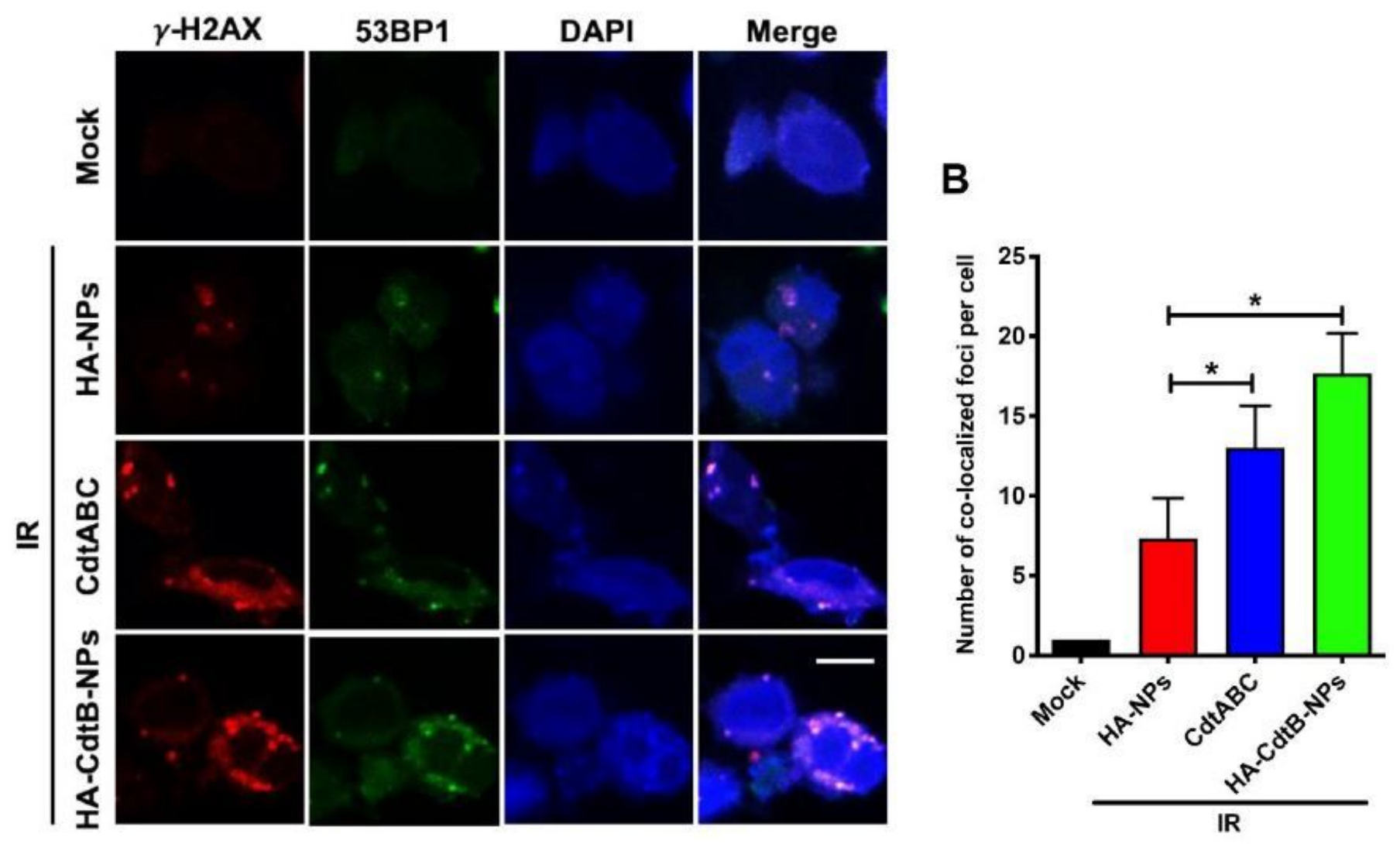

Figure 5

HA-CdtB-NPs sensitize PCa cells to radiation by promoting DSB. PC3-KD cells were mock-treated or exposed to IR (2 Gy) then incubated with $100 \mathrm{nM}$ HA-NPs, CDT holotoxin, and HA-CdtB-NPs for $24 \mathrm{~h}$. (A) Fluorescent immunostaining of $\mathrm{Y}-\mathrm{H} 2 \mathrm{AX}$ (red) and 53BP1 (green) was shown. DAPI (blue) was used as a tracer for cell nucleus. Scale, $10 \mu \mathrm{m}$. (B) The foci of $\mathrm{y}-\mathrm{H} 2 \mathrm{AX}$ and 53BP1 colocalization in the nuclei were counted. Statistical significance was evaluated using one-way ANOVA with post hoc test $(*, p<0.05)$. 
A

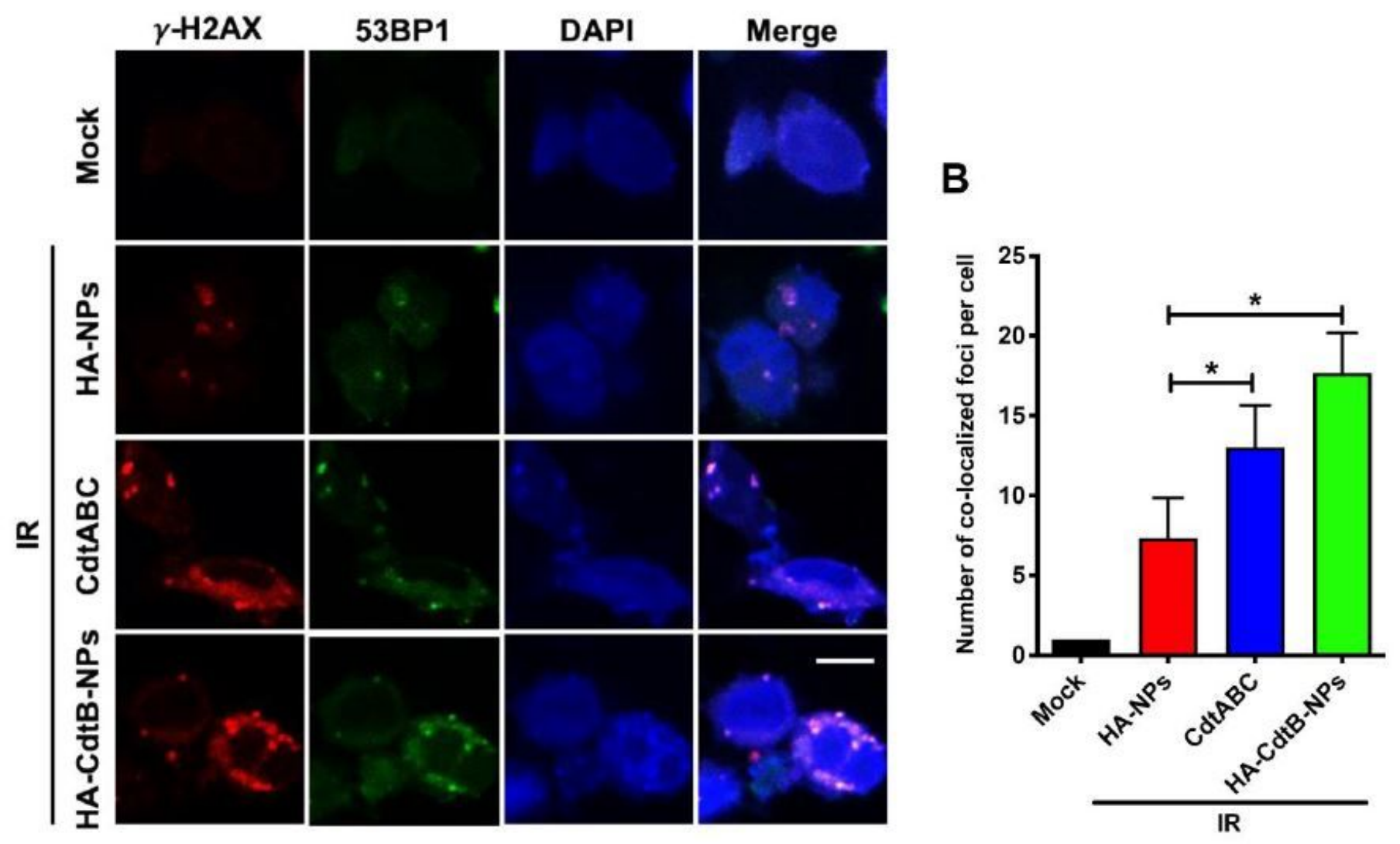

Figure 5

HA-CdtB-NPs sensitize PCa cells to radiation by promoting DSB. PC3-KD cells were mock-treated or exposed to IR (2 Gy) then incubated with $100 \mathrm{nM}$ HA-NPs, CDT holotoxin, and HA-CdtB-NPs for $24 \mathrm{~h}$. (A) Fluorescent immunostaining of $\mathrm{Y}-\mathrm{H} 2 \mathrm{AX}$ (red) and 53BP1 (green) was shown. DAPI (blue) was used as a tracer for cell nucleus. Scale, $10 \mu \mathrm{m}$. (B) The foci of $\mathrm{y}-\mathrm{H} 2 \mathrm{AX}$ and 53BP1 colocalization in the nuclei were counted. Statistical significance was evaluated using one-way ANOVA with post hoc test $(*, p<0.05)$. 
A

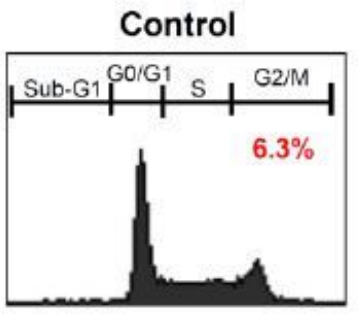

HA-CdtB-NPs

$50 \mathrm{nM}$

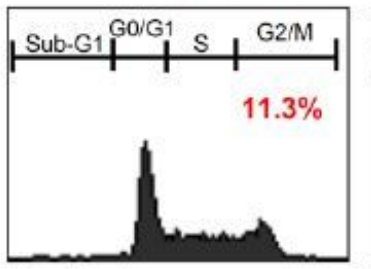

ICRF-193

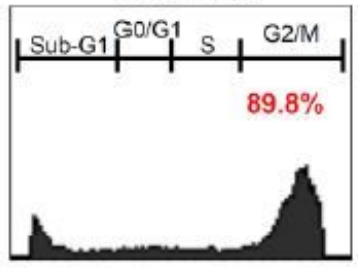

HA-CdtB-NPs $100 \mathrm{nM}$

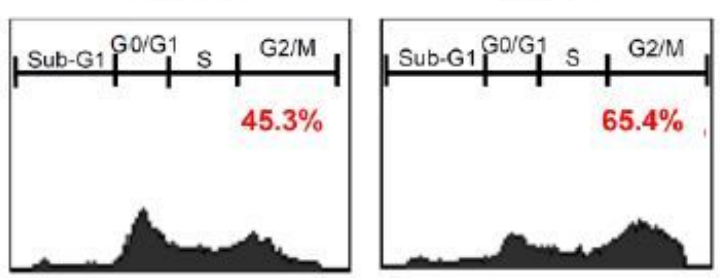

HA-NPs

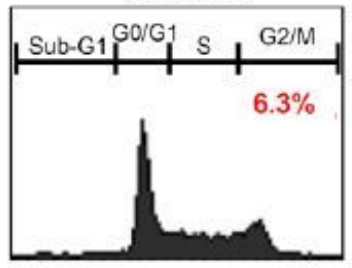

HA-CdtB-NPs $200 \mathrm{nM}$
B

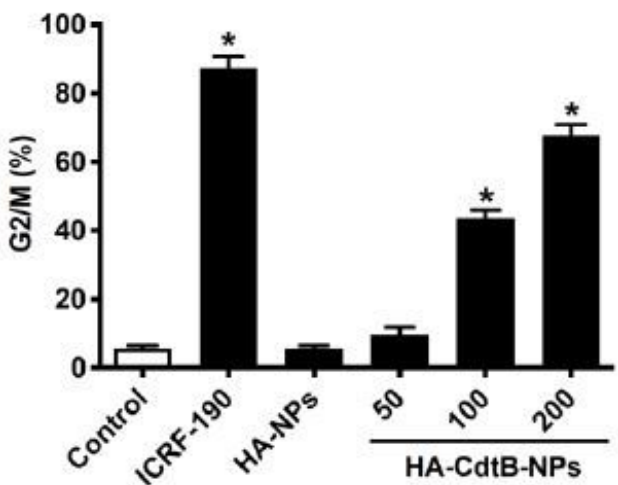

Figure 6

HA-CdtB-NPs induce cell cycle arrest and apoptosis. (A) PC3-KD cells were mock-treated, exposed to ICRF193 or HA-CdtB-NPs $(50,100$ and $200 \mathrm{nM})$ and incubated for $48 \mathrm{~h}$. Cell cycle distribution based on DNA content was analyzed using flow cytometry. (B) The percentage of cells at G2/M phase were calculated. Statistical significance was evaluated using one-way ANOVA with post hoc test $(*, p<0.05)$.

A

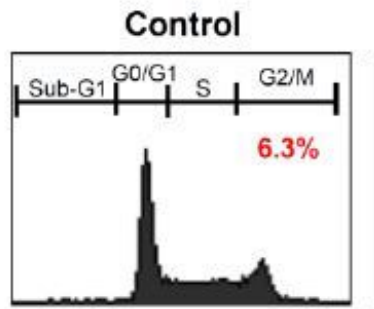

HA-CdtB-NPs $50 \mathrm{nM}$

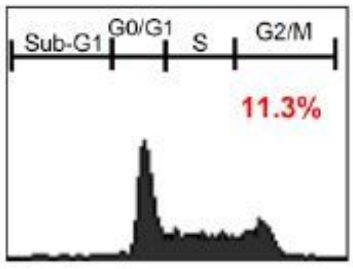

ICRF-193

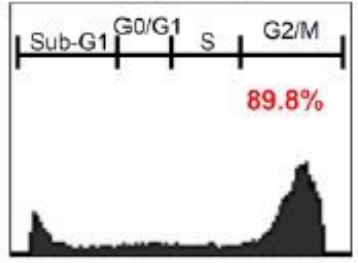

HA-CdtB-NPs $100 \mathrm{nM}$

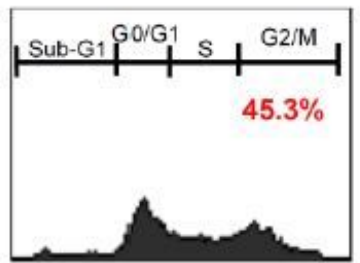

HA-NPs

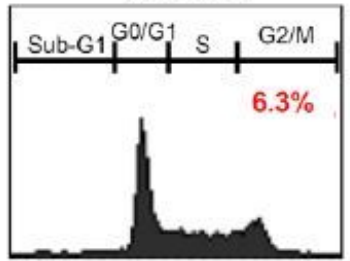

HA-CdtB-NPs $200 \mathrm{nM}$

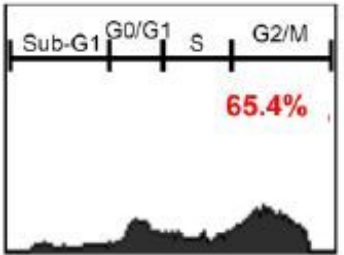

B

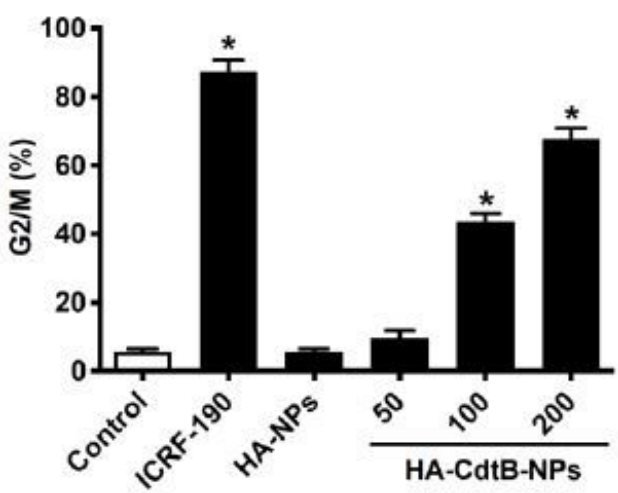

Figure 6 
HA-CdtB-NPs induce cell cycle arrest and apoptosis. (A) PC3-KD cells were mock-treated, exposed to ICRF193 or HA-CdtB-NPs $(50,100$ and $200 \mathrm{nM})$ and incubated for $48 \mathrm{~h}$. Cell cycle distribution based on DNA content was analyzed using flow cytometry. (B) The percentage of cells at G2/M phase were calculated. Statistical significance was evaluated using one-way ANOVA with post hoc test $(*, p<0.05)$.

\section{A}

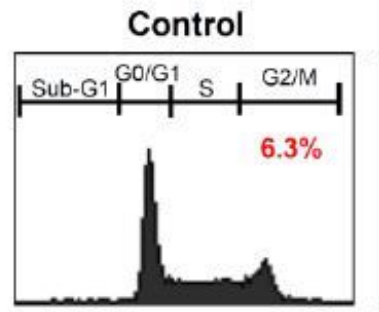

HA-CdtB-NPs $50 \mathrm{nM}$

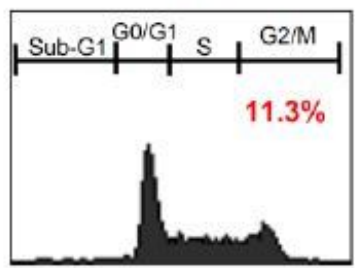

ICRF-193

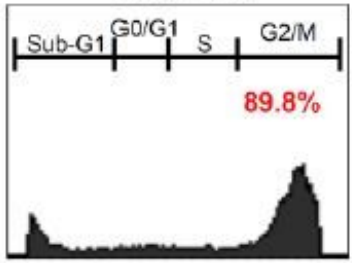

HA-CdtB-NPs $100 \mathrm{nM}$

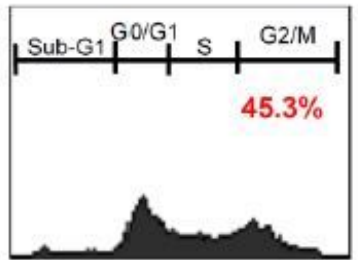

HA-NPS

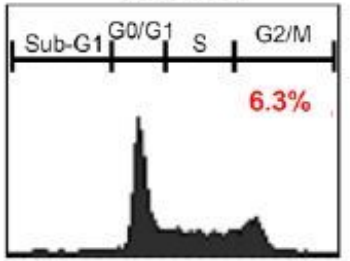

HA-CdtB-NPs $200 \mathrm{nM}$

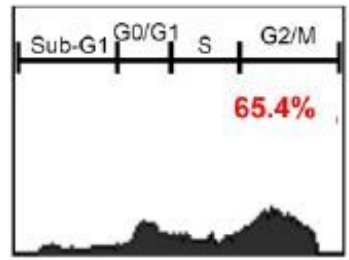

B

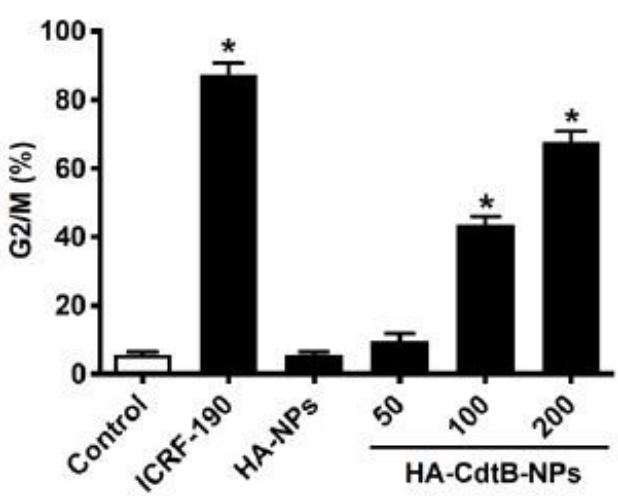

Figure 6

HA-CdtB-NPs induce cell cycle arrest and apoptosis. (A) PC3-KD cells were mock-treated, exposed to ICRF193 or HA-CdtB-NPs $(50,100$ and $200 \mathrm{nM})$ and incubated for $48 \mathrm{~h}$. Cell cycle distribution based on DNA content was analyzed using flow cytometry. (B) The percentage of cells at G2/M phase were calculated. Statistical significance was evaluated using one-way ANOVA with post hoc test $(*, p<0.05)$. 
A

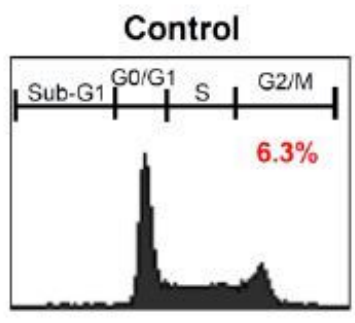

HA-CdtB-NPs $50 \mathrm{nM}$

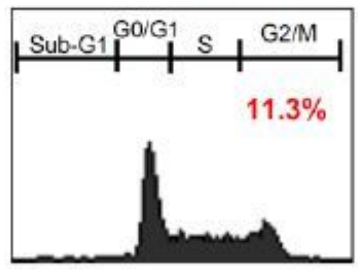

ICRF-193

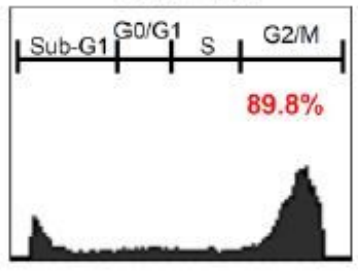

HA-CdtB-NPs $100 \mathrm{nM}$

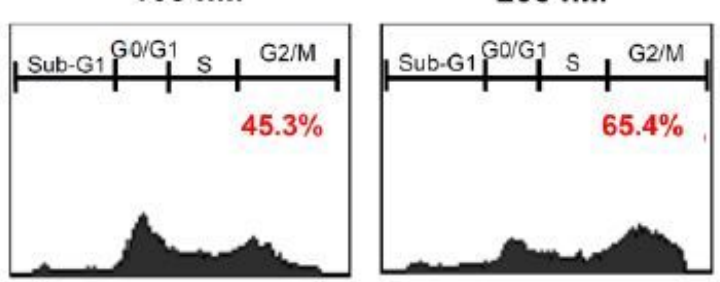

HA-NPs

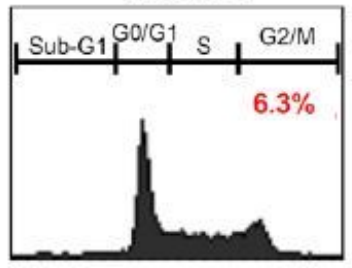

HA-CdtB-NPs $200 \mathrm{nM}$
B

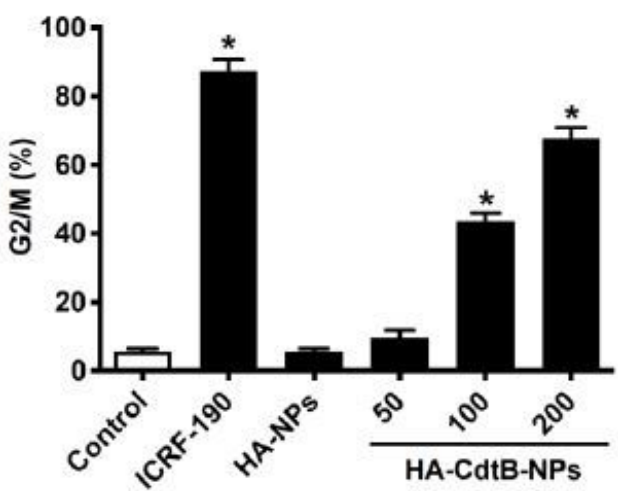

Figure 6

HA-CdtB-NPs induce cell cycle arrest and apoptosis. (A) PC3-KD cells were mock-treated, exposed to ICRF193 or HA-CdtB-NPs $(50,100$ and $200 \mathrm{nM})$ and incubated for $48 \mathrm{~h}$. Cell cycle distribution based on DNA content was analyzed using flow cytometry. (B) The percentage of cells at G2/M phase were calculated. Statistical significance was evaluated using one-way ANOVA with post hoc test $(*, p<0.05)$. 


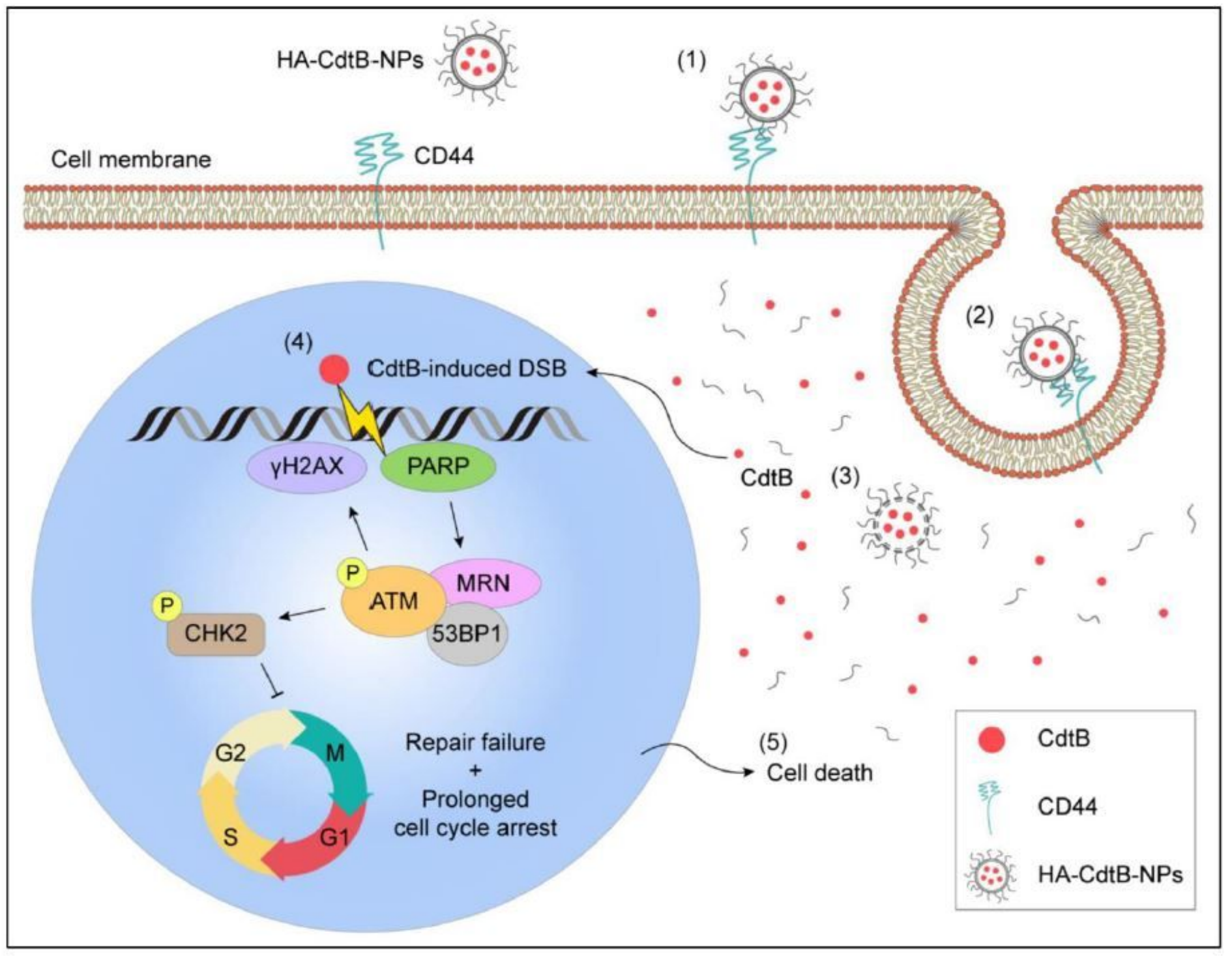

\section{Figure 7}

The effects of HA-CdtB-NPs on radioresistant PCa cells. (1) HA-based nanoparticles specifically bind to CD44 on the cell membrane and (2) the encapsulated CdtB is delivered into PCa cells through endocytosis. (3) With nuclear translocation signal (NLS), CdtB enters the nucleus and (4) exerts its DNase activity to effectively induce DSBs, leading to cell cycle arrest. (5) As the cancer cells fail to repair the extensive DNA damage and experiences prolonged cell cycle arrest, it is sentenced to death. This study unveils the mechanism behind the radiosensitivity enhancement in PCa cells promoted by HA-CdtB-NPs, and thus provides a promising target-specific agent for the development of a new therapy against radioresistant $\mathrm{PCa}$. 


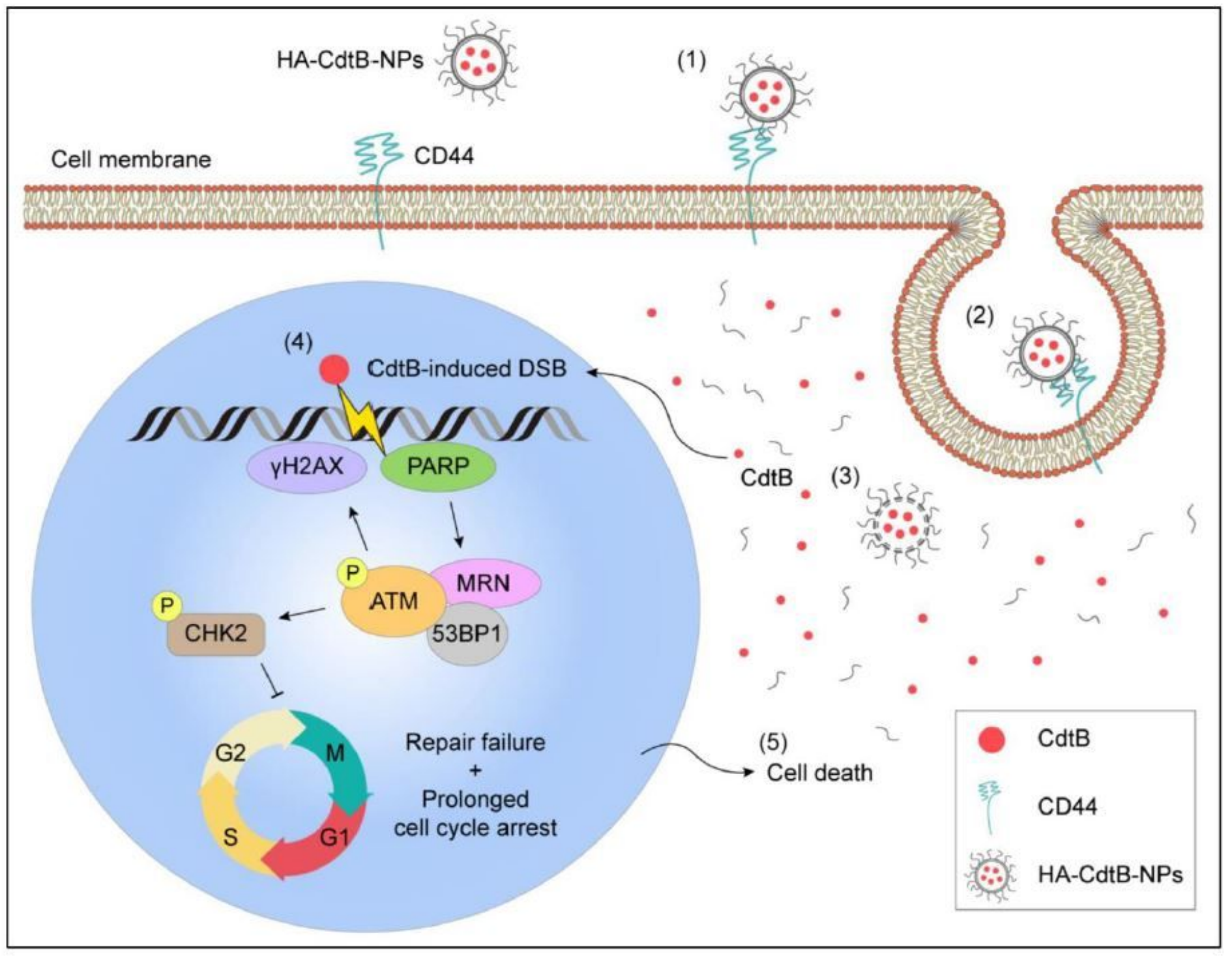

\section{Figure 7}

The effects of HA-CdtB-NPs on radioresistant PCa cells. (1) HA-based nanoparticles specifically bind to CD44 on the cell membrane and (2) the encapsulated CdtB is delivered into PCa cells through endocytosis. (3) With nuclear translocation signal (NLS), CdtB enters the nucleus and (4) exerts its DNase activity to effectively induce DSBs, leading to cell cycle arrest. (5) As the cancer cells fail to repair the extensive DNA damage and experiences prolonged cell cycle arrest, it is sentenced to death. This study unveils the mechanism behind the radiosensitivity enhancement in PCa cells promoted by HA-CdtB-NPs, and thus provides a promising target-specific agent for the development of a new therapy against radioresistant $\mathrm{PCa}$. 


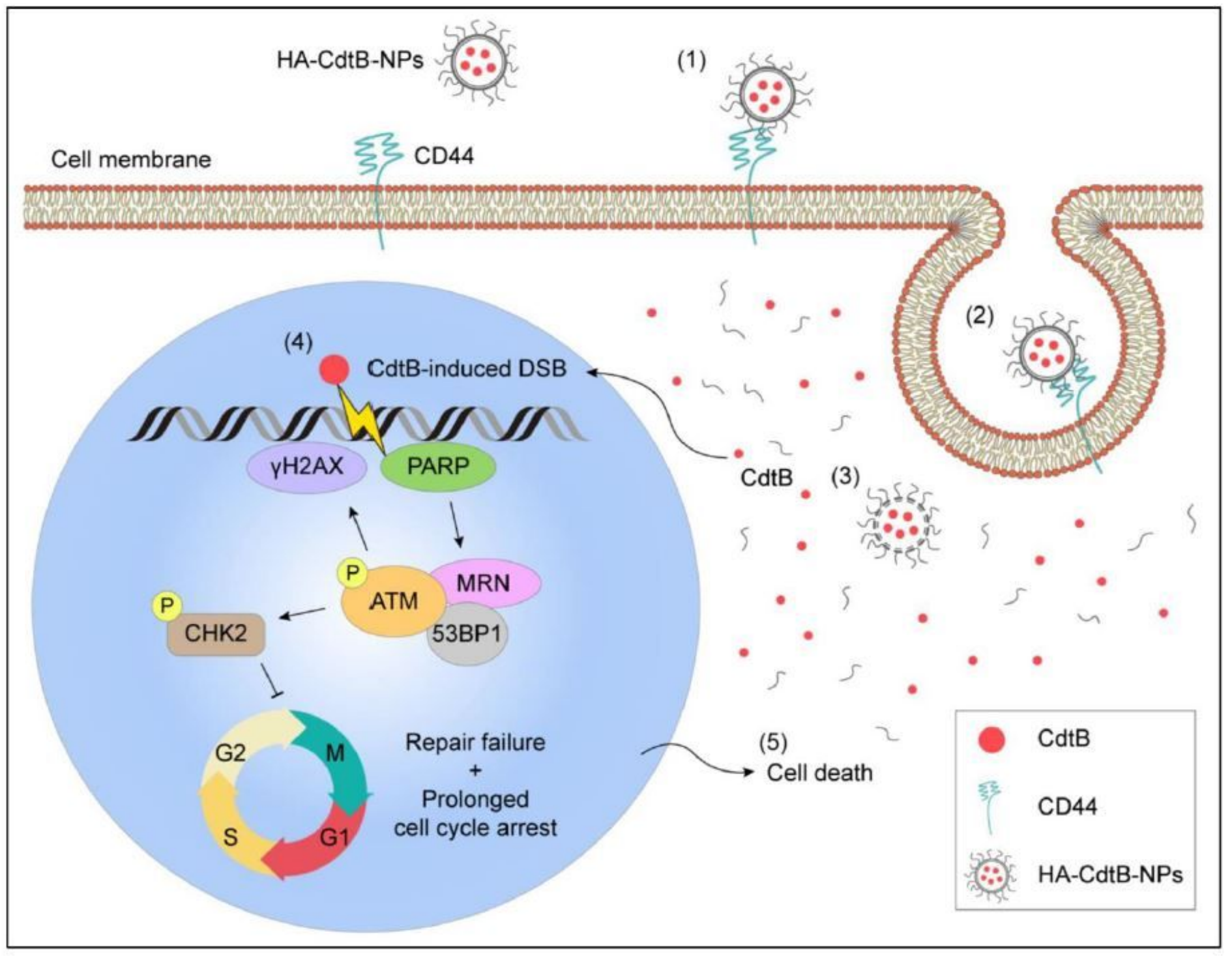

\section{Figure 7}

The effects of HA-CdtB-NPs on radioresistant PCa cells. (1) HA-based nanoparticles specifically bind to CD44 on the cell membrane and (2) the encapsulated CdtB is delivered into PCa cells through endocytosis. (3) With nuclear translocation signal (NLS), CdtB enters the nucleus and (4) exerts its DNase activity to effectively induce DSBs, leading to cell cycle arrest. (5) As the cancer cells fail to repair the extensive DNA damage and experiences prolonged cell cycle arrest, it is sentenced to death. This study unveils the mechanism behind the radiosensitivity enhancement in PCa cells promoted by HA-CdtB-NPs, and thus provides a promising target-specific agent for the development of a new therapy against radioresistant $\mathrm{PCa}$. 


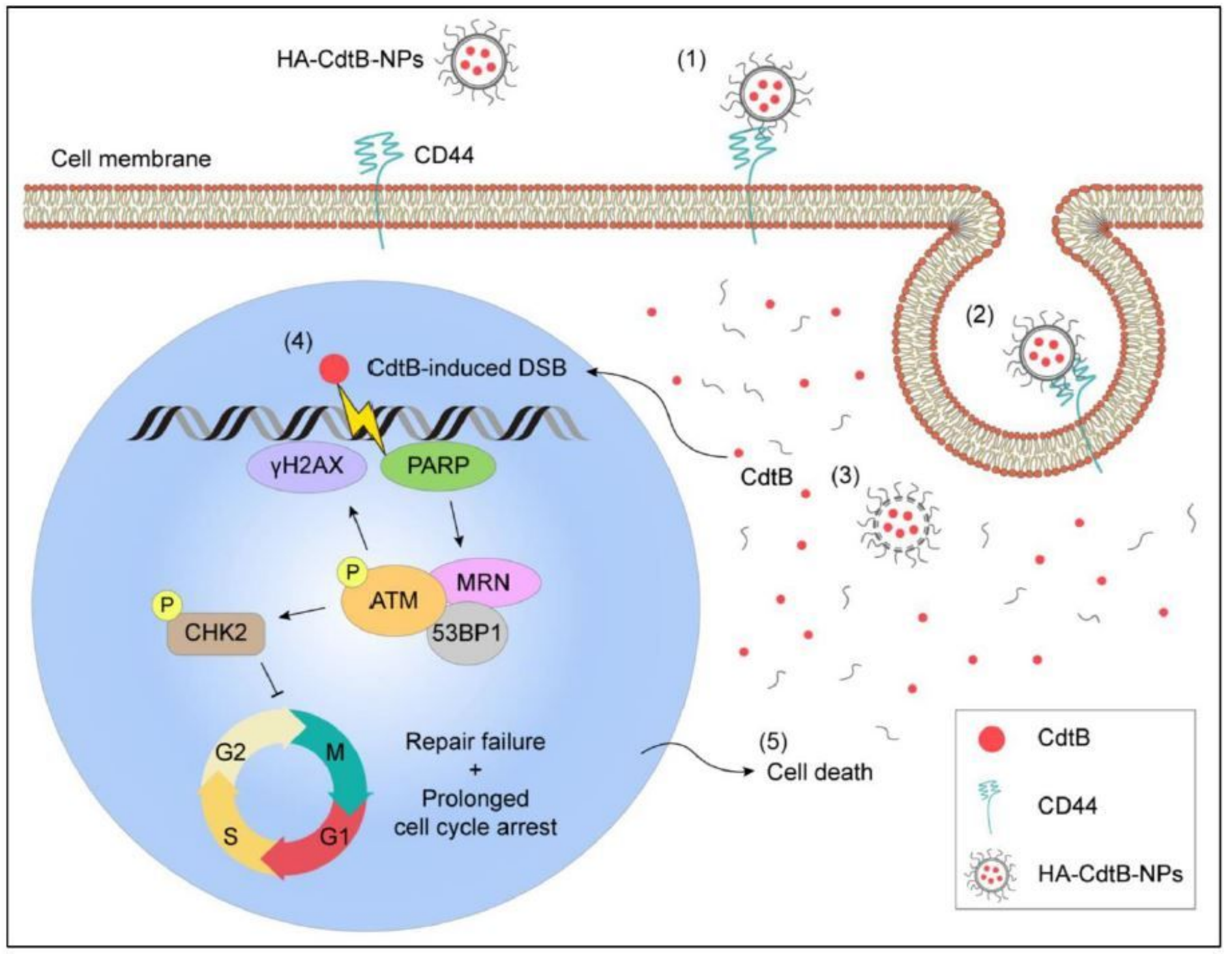

\section{Figure 7}

The effects of HA-CdtB-NPs on radioresistant PCa cells. (1) HA-based nanoparticles specifically bind to CD44 on the cell membrane and (2) the encapsulated CdtB is delivered into PCa cells through endocytosis. (3) With nuclear translocation signal (NLS), CdtB enters the nucleus and (4) exerts its DNase activity to effectively induce DSBs, leading to cell cycle arrest. (5) As the cancer cells fail to repair the extensive DNA damage and experiences prolonged cell cycle arrest, it is sentenced to death. This study unveils the mechanism behind the radiosensitivity enhancement in PCa cells promoted by HA-CdtB-NPs, and thus provides a promising target-specific agent for the development of a new therapy against radioresistant $\mathrm{PCa}$.

\section{Supplementary Files}

This is a list of supplementary files associated with this preprint. Click to download. 
- GraphicalAbstract.pdf

- GraphicalAbstract.pdf

- GraphicalAbstract.pdf

- GraphicalAbstract.pdf

- Supplementaryinformation.pdf

- Supplementaryinformation.pdf

- Supplementaryinformation.pdf

- Supplementaryinformation.pdf 